\title{
EFFECT OF OMEGA 3 POLYUNSATURATED FATTY ACIDS (PUFAS) ON MARKERS OF INFLAMMATION IN YOUNG HORSES IN TRAINING
}

\author{
A Thesis \\ by \\ JESSICA LAUREN LUCIA
}

\begin{abstract}
Submitted to the Office of Graduate Studies of Texas A\&M University

in partial fulfillment of the requirements for the degree of

MASTER OF SCIENCE
\end{abstract}

December 2009

Major Subject: Animal Science 


\title{
EFFECT OF OMEGA 3 POLYUNSATURATED FATTY ACIDS (PUFAS) ON MARKERS OF INFLAMMATION IN YOUNG HORSES IN TRAINING
}

\author{
A Thesis \\ by \\ JESSICA LAUREN LUCIA
}

\begin{abstract}
Submitted to the Office of Graduate Studies of Texas A\&M University

in partial fulfillment of the requirements for the degree of

MASTER OF SCIENCE
\end{abstract}

Approved by:

Chair of Committee, Josie Coverdale

Committee Members, Carolyn Arnold

Clifford Honnas

Brett Scott

Head of Department, Gary Acuff

December 2009

Major Subject: Animal Science 


\begin{abstract}
Effect of Omega 3 Polyunsaturated Fatty Acids (PUFAS) on Markers of Inflammation in Young Horses in Training.

(December 2009)

Jessica Lauren Lucia, B.S., Texas A\&M University

Chair of Advisory Committee: Dr. Josie Coverdale
\end{abstract}

Sixteen horses ( 2 to $4 \mathrm{yr} ; 357$ to $439 \mathrm{~kg} \mathrm{BW}$ ) were utilized in a randomized complete block design for a 140 d trial to determine effect of omega 3 PUFAs (n-3) supplementation on markers of inflammation in young horses in training. Horses were fed treatments consisting of a control diet $(\mathrm{n}=8)$ fed at $1 \% \mathrm{BW}($ as fed) or a treatment $\operatorname{diet}(\mathrm{n}=8)$ of concentrate fed at $0.75 \% \mathrm{BW}$ (as fed) and $350 \mathrm{~g}$ of a marine $\mathrm{n}-3$ supplement formulated to provide $15 \mathrm{~g}$ of eicosapentaenoic acid (EPA) and $20 \mathrm{~g}$ of docosahexaenoic acid (DHA). Body weight and body condition scores (BCS) were obtained biweekly and concentrate adjusted accordingly.

Horses were exercised $5 \mathrm{~d} / \mathrm{wk}$ by students in an equine training course. Type of activity and duration was monitored, along with heart rate to quantify workload. Exercise protocol was divided into 2 phases: phase I (d 0 to110) consisted of ground work and early training under saddle, and phase II (d 111 to 140) consisted of advance maneuvers and moderate workload. 
Synovial fluid was obtained from right radial carpal joint by arthrocentesis every $28 \mathrm{~d}$ and was analyzed for white blood cell count (WBC), total protein (TP), and specific gravity (SG). Serum concentrations of carboxypeptide type II collagen (CPII) and chondroitin sulfate 846 (CS-846) were analyzed by ELISA kits. Dietary treatment did not affect synovial WBC, TP, or SG. Also, concentrations of WBC and TP also did not differ over time. SG increased over time $(\mathrm{P}<0.001)$ as horses moved from phase I to phase II of the trial. Dietary treatment did not influence concentrations of CPII or CS846. CS-846 tended to increase over time $(P=0.09)$ and CPII concentrations also increased $(\mathrm{P}<0.001)$ in response to changes in exercise. Furthermore, all horses gained BW and BCS throughout the trial $(\mathrm{P}<0.001)$, but values were not influenced by treatment. This data indicates further studies are needed to determine the efficacy of n-3 supplementation as a preventative measure against development of osteoarthritis. 


\section{DEDICATION}

For Mema and Papa 


\section{ACKNOWLEDGEMENTS}

I would like to thank my committee chair, Dr. Josie Coverdale, and my committee members, Dr. Arnold, Dr. Honnas, and Dr. Scott for their guidance and support throughout the course of this research. I would also like to extend my appreciation to Dr. Tom Welsh and Nicole Burdick for their time, assistance, and use of equipment for the completion of laboratory analysis. I also want to thank my fellow graduate students for their willingness to work and devote long hours in order to complete this project. Finally, thanks to my mom and dad for their encouragement, continued love, support, patience, and help over the past two years. I would also like to thank my loving fiancé for his long hours and assistance with the daily routine at the barn. Words cannot express the gratitude and thanks they all deserve. 
TABLE OF CONTENTS

Page

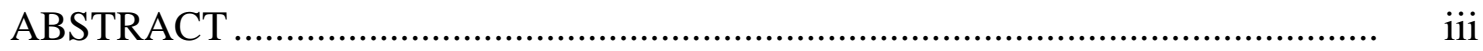

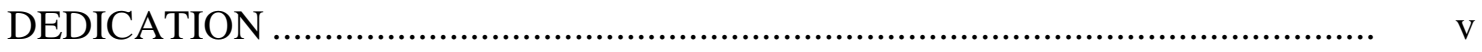

ACKNOWLEDGEMENTS …………………............................................ vi

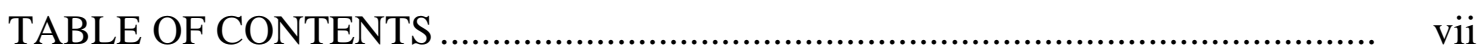

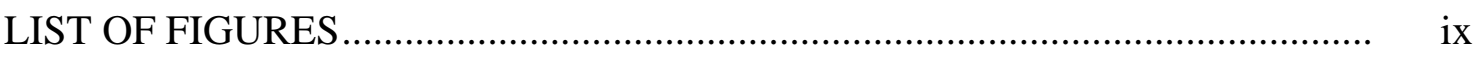

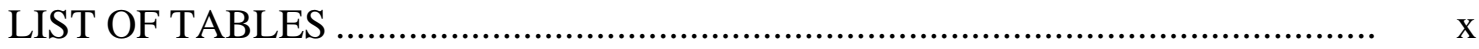

CHAPTER

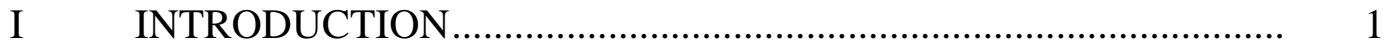

II REVIEW OF THE LITERATURE ................................................. 3

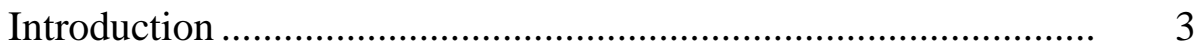

Dietary Lipids ........................................................................ 5

Lipids in the Equine Diet ..................................................... 5

Essential Fatty Acids .................................................................. 6

Cyclooxygenase Pathway ............................................................ 7

Supplemental Forms of n-3 PUFAs .......................................... 10

Dietary n-6 to n-3 Ratio ........................................................... 11

Health Mitigation of Omega 3 Supplementation ........................... 14

Coronary Heart Disease ............................................................ 14

Exercise Tolerance ………................................................... 15

Joint Disease ........................................................................ 16

Impact of Equine Joint Disease ................................................. 19

Bone and Cartilage Response to Exercise ...................................... 21

Bone ....................................................................... 22

Articular Cartilage ..................................................................... 23

Anabolic Markers Relative to Joint Health ..................................... 29

Inflammatory Markers.............................................................. 31

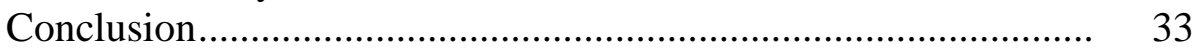


CHAPTER Page

III $\quad$ MATERIALS AND METHODS …............................................ 35

Horses and Dietary Treatments ............................................ 35

Exercise Protocol.................................................................... 37

Phase I: Ground Work and Early Training.............................. 37

Phase II: Advanced Maneuvers and Moderate Workload.......... 38

Sample Collection ................................................................ 39

Sample Analysis ................................................................. 40

Gross Energy Determination .................................................... 40

Synovial Fluid and Serum Analysis ..................................... 41

Statistical Analysis ............................................................ 43

IV $\quad$ RESULTS AND DISCUSSION …........................................ 44

Body Weight and Body Condition Score .................................. 44

Serum Anabolic Markers ........................................................... 46

Synovial Fluid Inflammatory Markers ..................................... 50

V SUMMARY ........................................................................ 53

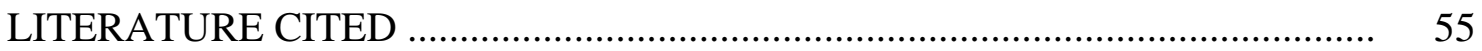

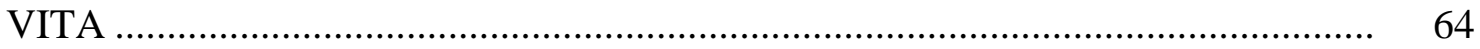




\section{LIST OF FIGURES}

Page

Figure 1 The anabolic pathway of essential fatty acids

Figure 2 Cyclooxygenase pathway of arachidonic acid (AA) metabolism.....

Figure 3 Structure of type II collagen

Figure 4 Changes in BW (kg) over time (d) beginning with phase I (d 0 to d 110) consisting of groundwork and early training to phase II (d 111-140) representing moderate workload and application of advanced maneuvers in horses receiving a pelleted concentrate with (Treatment) or without (Control) supplementation of a marine based omega 3 product

Figure 5 Changes in body condition score (BCS) over time (d) beginning with phase I ( 0 to d 110) consisting of ground work and early training to phase II (d 111-140) representing moderate workload and application of advanced maneuvers in horses receiving a pelleted concentrate with (Treatment) or without (Control) supplementation of a marine based omega 3 product

Figure 6 Serum concentrations $(\mathrm{ng} / \mathrm{mL})$ of the carboxypeptide of type II collagen (CPII) over time (d) beginning with phase I (d 0 to 110) consisting of ground work and early training to phase II (d 111-140) representing moderate workload and application of advanced maneuvers in horses receiving a pelleted concentrate with (Treatment) or without (Control) supplementation of a marine based omega 3 product

Figure 7 Serum concentrations $(\mathrm{ng} / \mathrm{mL})$ of the epitope chondroitin sulfate 846 (CS-846) over time (d) beginning with phase I (d 0 to 110) consisting of ground work and early training to phase II (d 111 to 140) representing moderate workload and application of advanced maneuvers in horses receiving a pelleted concentrate with (Treatment) or without (Control) supplementation of a marine based product. 


\section{LIST OF TABLES}

Page

Table 1 Gross energy content (Mcal/kg) of diet components .....

Table 2 Unsaturated fatty acid profile of concentrate, coastal bermudagrass hay, and marine based n-3 supplement

Table 3 Heart rates expressed in beats per minute (bpm) of randomly selected horses in response to exercise

Table 4 Least square treatment means of white blood cell count (WBC), total protein (TP), and specific gravity (SG) in synovial fluid of horses with (Treatment) or without (Control) omega 3 supplementation 


\section{CHAPTER I}

\section{INTRODUCTION}

Osteoarthritis (OA) is one of the most significant causes of early retirement of many young equine athletes (Todhunter and Lust, 1990). Bone and soft tissue adaptation occurs during early training. During adaptation, young horses undergo repeated stress that can result in the production of inflammatory eicosanoids. These eicosanoids are synthesized by the cyclooxygenase pathway $(\mathrm{COX})$ and are responsible for breakdown of articular cartilage (Bertone et al., 2001). Omega 3 (n-3) fatty acids such as eicosapentaenoic acid (EPA) and docosahexaenoic (DHA) acid compete with omega 6 (n-6) fatty acids during metabolism to produce less potent inflammatory compounds. A previous study utilizing arthritic horses demonstrated the effectiveness of n-3 supplementation to mitigate inflammation (Manhart et al., 2009).

Determining the effectiveness of $\mathrm{n}-3$ supplementation as a preventative measure against OA in young horses poses great difficulty. However, markers of cartilage metabolism may provide insight to the effectiveness of n-3 supplementation. Carboxypeptide of type II collagen (CPII) and chondroitin sulfate 846 (CS-846) concentrations can be determined as synthesis of proteoglycans and type II collagen in attempts to repair cartilage. These collagenases are released at higher concentrations into synovial fluid and blood (Frisbie et al., 1999). Synovial fluid

This thesis follows the style of Journal of Animal Science. 
concentrations of white blood cells (WBC), specific gravity (SG) and total protein (TP) have also been utilized as markers of inflammation and increase in response to articular cartilage damage (Bertone et al., 2001).

It is evident that joint damage occurring early in a horse's athletic career may reflect the incidence of OA later in life. Determining a preventative strategy that can decrease articular cartilage damage during early training is valuable. The objective of this study was to determine the effect of n-3 fatty acid supplementation on markers of joint inflammation in young horses in training. 


\section{CHAPTER II}

\section{REVIEW OF THE LITERATURE}

\section{Introduction}

Inflammatory joint disorders are considered to be a common cause of lameness in horses, as well as, the leading cause for early retirement of athletic horses (Jeffcoat et al., 1982). Young horses have the highest rate of cartilage formation and can easily repair and replace damaged joint tissue (Brama et al., 2000). However, during early training and exercise, horses are subjected to large mechanical forces that can potentially lead to an imbalance between the synthesis and degradation of articular cartilage. The enzymatic breakdown of articular cartilage results in the release of inflammatory mediators including cytokines and eicosanoids that are synthesized through the cyclooxygenase $(\mathrm{COX})$ pathway.

Both n-3 and n-6 PUFAs are found within the equine diet and actively participate in the COX pathway. Young performance horses often receive fat supplemented diets in the form of vegetable oils (primarily corn), increasing caloric density of the diet and lowering heat increment (Scott et al., 1992). However, this results in an increase in the dietary ratio of n-6 to n-3 fatty acids. When n-6 fatty acids enter into the COX pathway, they produce the potent inflammatory compound, arachidonic acid (AA). In contrast to n-6 fatty acids, marine oils are high in n-3 fatty acids, in particular eicosapentaenoic acid (EPA) and docosahexaenoic (DHA), and are known to produce less potent inflammatory compounds, with some compounds being considered anti-inflammatory (Calder, 2002). Dietary addition of n-3 fatty acids compete with n- 6 fatty acids for incorporation into 
cellular membranes and therefore could potentially decrease the inflammatory response that is associated with degenerative joint disease.

Trials with humans have demonstrated the efficacy of n-3 fatty acids in the reduction of anti-inflammatory lipids (resolvins and protectins) as well as, reduction in joint pain associated with OA (Calder, 2002). Determining the effectiveness of $n-3$ supplementation in young performance horses poses great difficulty. Often, performance characteristics are difficult to measure objectively and may not be sensitive enough to detect the early stages of joint damage. However, direct markers of cartilage metabolism may provide insight on the efficacy of n-3 fatty acid supplementation. Carboxypeptide of type II collagen (CPII) and chondroitin sulfate-846 (CS-846) have been shown to increase with osteochondral damage in response to exercise in nonarthritic horses (Frisbie et al., 2008). These collagenases are released at higher concentrations into the blood as well as synovial fluid and are a reflection of the early stages of osteochondral fragmentation (Frisbie et al., 2008). Furthermore, indirect markers including WBC, TP, and SG, have been used as markers of inflammation and have been shown to increase in response to articular cartilage damage (Calder, 2002). Biochemical markers, both direct and indirect, can potentially be utilized as an effective means to evaluate n-3 supplementation in young horses in training.

A previous study utilizing arthritic horses demonstrated the effectiveness of $n-3$ fatty acid supplementation to mitigate inflammation in horses previously diagnosed with OA (Manhart et al., 2009). However, little information exists concerning supplementation to juvenile horses in training. Therefore, determining a preventative 
strategy that can inhibit or decrease articular cartilage damage during early training would be valuable.

\section{Dietary Lipids}

Dietary lipids exist in the form of triglycerides, consisting of three fatty acid molecules attached to a glycerol backbone. Triglycerides exist as fats or oils at room temperature based upon the components and physical properties of the individual fatty acids. Fatty acids are described by the number of carbons and double bonds they contain, as well as by the position of the first double bond in relation to the methyl or the omega end of the molecule (Vance and Vance, 1991). Two major families of fatty acids that are characteristic of mammalian species are the n-6 and the n-3 PUFAs. The n-3 and n-6 PUFAs are defined by the position of the first double bond that is closest to the terminal methyl group of the fatty acid molecule (Vance and Vance, 1991). For example, within the n- 6 family, the first double bond occurs between the sixth and seventh carbon from the methyl end of the molecule, whereas in the $n-3$ family the first double bond occurs between the third and fourth carbon.

\section{Lipids in the Equine Diet}

Lipids are generally utilized in the equine diet in order to increase the energy density and/or substitute for rapidly fermentable carbohydrates in the form of cereal grains (Scott et al., 1992). Meyers et al. (1989) concluded that increasing the energy density of feeds by adding dietary fat may allow a reduction in total feed intake required to meet energy requirements, which could reduce the chance of fermentation-related digestive disorders in performance horses. Supplemental animal and vegetable fats have 
been utilized in the equine diet, in the form of vegetable oils, bi-product feeds (stabilized rice bran and wheat germ), along with medium chain fatty acids such as fish oil (menhaden). However, the use of vegetable oils, primarily corn oil, is the most prevalent due to its superior palatability (Holland et al., 1997). The benefits of feeding fat supplemented diets to performance horses are well documented, including increased caloric density, lower heat increment, and a glycogen sparing effect (Webb et al., 1987; Meyers et al., 1989; Julen et al., 1995), although in more recent years, interest has been placed on the fatty acid composition of the diet.

Essential Fatty Acids

Mammals lack the enzymes required for induction of double bonds in fatty acids greater than 9 carbons in length (Nakamura and Nara, 2002). Therefore, these basic structures cannot be synthesized de novo and must be supplied by the diet. Mammals cannot convert oleic acid into linoleic acid, and they cannot convert linoleic acid into $\alpha$ linolenic acid, because the desaturases required for these reactions are found only in plants. The shorter chain essential fatty acids, including linoleic and $\alpha$-linolenic acid, serve as initial precursors for synthesis of longer chain PUFAs, which involves a series of desaturases and elongases in which 2 hydrogen atoms are removed to create a new double bond followed by the addition of 2 carbon atoms to lengthen the fatty acid chain (Marcel et al., 1968; Figure 1). 


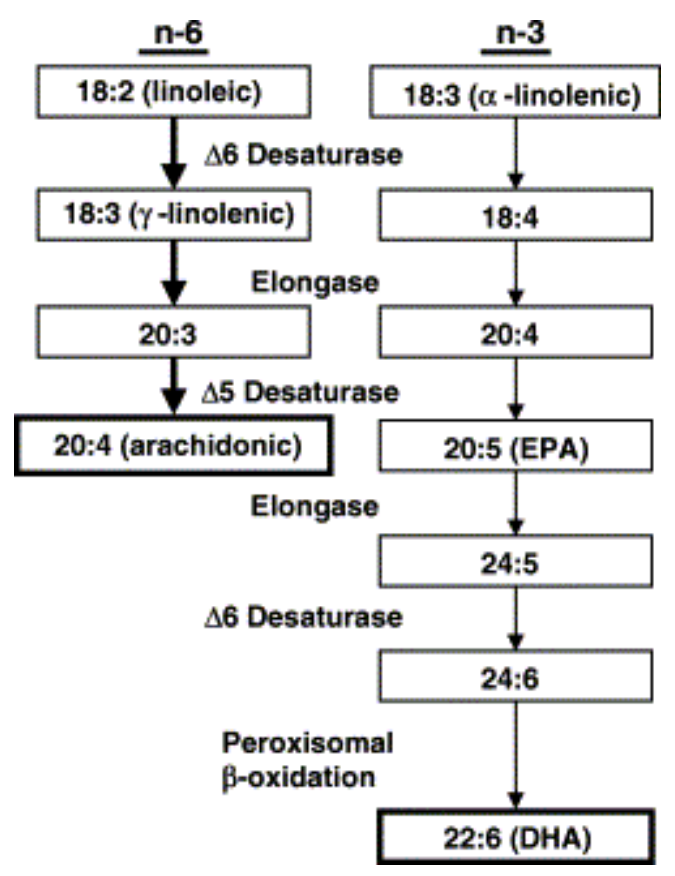

Figure 1. The anabolic pathway of essential fatty acids (Nakamura and Nara, 2002).

\section{Cyclooxygenase Pathway}

Long chain PUFAs have been recognized as substrates of various oxygenation reactions, many of which lead to important biologically active compounds (Vance and Vance, 1991). The essential fatty acid, linoleic acid, serves as a precursor for production of arachidonic acid (AA). Arachidonic acid, a common dietary n-6 fatty acid, is considered to be one of the most important PUFAs associated with membrane phospholipids, because the membranes of most cells contain higher concentrations of AA when compared to other eicosanoid precursors (Calder, 2002). Arachidonic acid is normally stored in membrane-bound phospholipids and is released by the action of phospholipases (Samuelsson, 1987). 
The enzymatic conversion of released AA into biologically active derivatives proceeds through one of several routes, including the COX pathway (Figure 2). Two isoforms of the COX pathway exist: COX-1 is a constitutive enzyme (always present in tissues); $\mathrm{COX}-2$ is induced in inflammatory cells as a result of stimulation, and is responsible for the markedly elevated production of eicosanoids and cytokines. These short-lived mediators derived from AA are responsible for modulating the intensity and duration of an inflammatory response, along with regulating various homeostatic functions (Calder, 2002).

In opposition, dietary $\alpha$-linolenic acid serves as a precursor to EPA and DHA (commonly found in marine oils) which are very effective in attenuating tissue AA levels and eicosanoid production. Many of the beneficial physiological effects of $n-3$ PUFAs have been linked to their ability to inhibit the AA cascade (Li et al., 1994). Also, EPA is considered to have a close homology with AA because they differ only by presence or absence of a double bond (Cleland et al., 2003). Both EPA and DHA compete with AA and other n-6 PUFAs for storage into the cell membrane and share the same receptors on target cells, and as a result, there is less substrate available for the synthesis of eicosanoids from AA (Calder, 2002). Also, EPA inhibits the release of AA by decreasing the release of phospholipase $\mathrm{A}_{2}$ and therefore decreases the production of AA (Obata et al., 1999). 


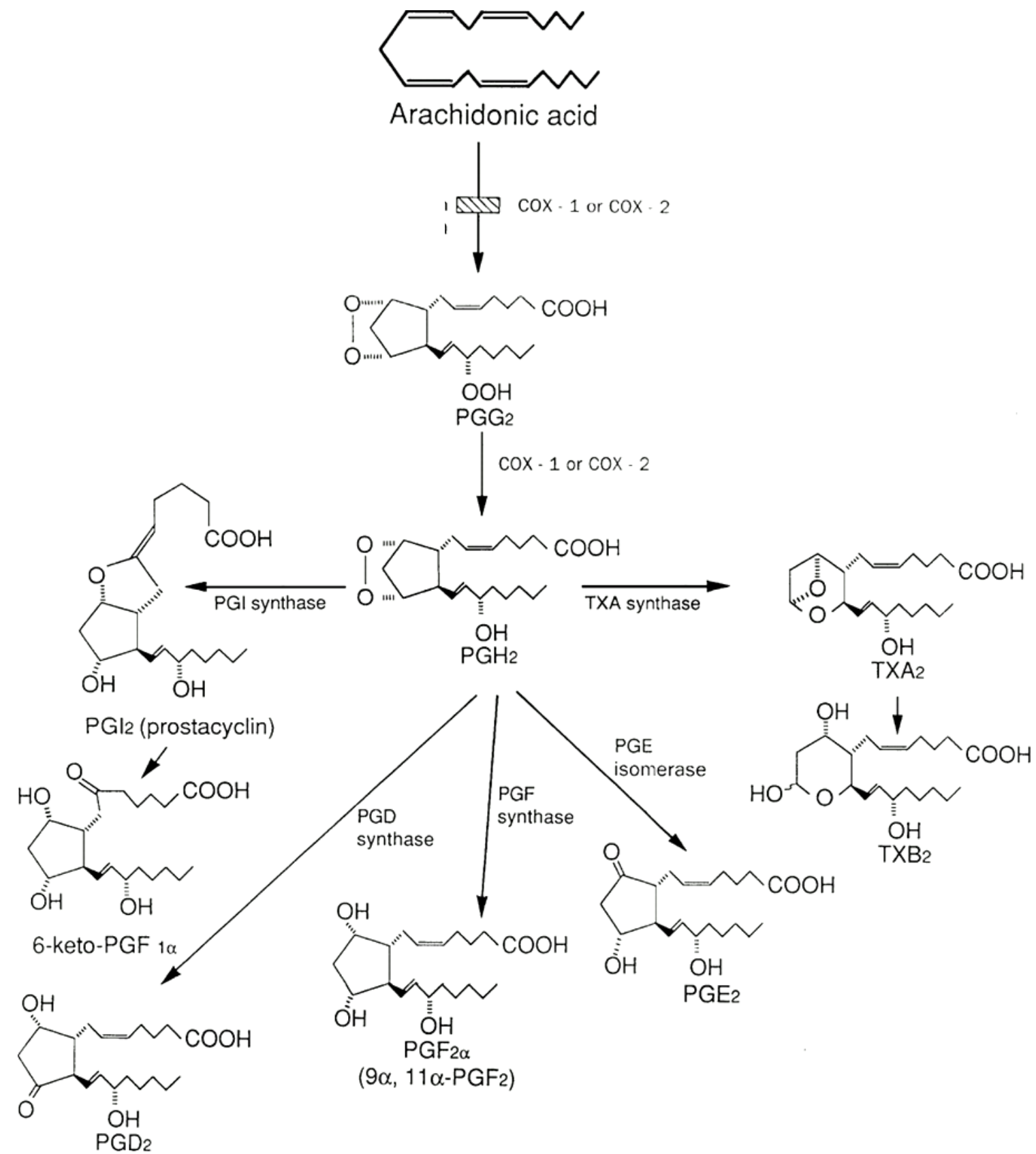

Figure 2. Cyclooxygenase pathway of arachidonic acid (AA) metabolism (Vane et al., 1998). 
In addition to inhibiting the metabolism of AA, EPA is able to act as a substrate for the COX pathway, giving rise to derivatives which have a structure different from those produced from AA (Calder, 2002). Dietary EPA is converted into an array of products that differ from AA derived eicosanoids by the presence of the $n-3$ double bond (Cleland et al., 2003). An example of differential inflammatory potencies of eicosanoids produced from AA and EPA is that of leukotriene $\mathrm{B}_{4}\left(\mathrm{LTB}_{4}\right)$ vs. leukotriene $\mathrm{B}_{5}\left(\mathrm{LTB}_{5}\right)$. Leukotriene $\mathrm{B}_{5}$ is approximately 10 -fold less potent when compared to $\mathrm{LTB}_{4}$ as a neutrophil chemoattractant on leukocytes, and on this basis can be considered less proinflammatory (Calder, 2002). The reduction in the generation of AA derived mediators has led to an interest in utilizing supplemental n-3 fatty acids for their anti-inflammatory potential.

Supplemental Forms of n-3 PUFAs

Linseed and flaxseed oil are naturally found in most diets or common supplements. Flaxseed oil is cold pressed and no solvents are used to extract additional oil from the meal. Linseed oil traditionally has been extracted from flaxseed under conditions of high temperatures and by petroleum extraction (Hansen et al., 2002). When raw linseed oil was administered to horses as a bolus, it was reported to cause anorexia or mild colic, however, a number of researchers have fed rations with $4-8 \%$ linseed or flaxseed oil without any apparent problems (Hansen et al., 2002).

Flaxseed or linseed oil provides a dietary source of $\alpha$-linolenic acid, although there is poor conversion efficiency from $\alpha$-linolenic acid to EPA and further into DHA. For example, human clinical trials have determined the proportion of $\alpha$-linolenic acid 
entering into the desaturation-elongation pathway that is converted into EPA is $<8$ to $10 \%$ and it has been approximated that the conversion into DHA is only $4 \%$ (Burdge, 2004). Additionally, Burdge (2004) reported the conversion rate of $\alpha$-linolenic acid to EPA can vary between $0.2 \%$ to $21 \%$ while the conversion of $\alpha$-linolenic to DHA varies between $0 \%$ to $9 \%$. A possible explanation for the poor conversion efficiency is due to the initial enzyme, $\Delta 6$ desaturase, which is considered to be a rate limiting step in many species, including horses (Cleland, 2003).

Flaxseed or linseed oil may also be an inappropriate source of n-3 PUFAs because of its relatively high proportion of linoleic acid content (Hansen et al., 2002). Hansen et al. (2002) investigated the effect of n-3 enriched diets in the form of flaxseed oil on plasma fatty acid concentrations and platelet aggregation in healthy horses. This study determined no marked decreases in AA or increases in DHA, indicating that flaxseed oil may have had a high percentage of n-6 fatty acids. This relatively high proportion of n-6 to n-3 ratio may have affected the biochemical activity of the n-3 fatty acids. Therefore, it may be more effective to provide a direct source of EPA and DHA, which are found in large amounts in marine based products, rather than products that are high in $\alpha$-linolenic acid such as flaxseed or linseed oil.

\section{Dietary n-6 to n-3 Ratio}

The concentrations of n-3 PUFAs in the cell membrane are dependent upon the intake of n-3 PUFAs in the diet, and therefore decreasing the n-6:n-3 ratio alters the composition of cellular membranes, by competitively inhibiting AA production, which leads to a decrease in the amount of cytokines and eicosanoids produced (Simopoulos, 
2002). In humans, excessive amounts of n-6 PUFAs and a very high n-6:n-3 ratio, as found in today's diets, promotes the pathogenesis of many diseases, including cardiovascular disease, cancer, and inflammatory diseases. Increased amounts of n-3 fatty acids (or a lower n-6:n-3 ratio), exert suppressive effects on the production of inflammatory mediators (Simopoulos, 2002). In the prevention of cardiovascular disease, a ratio of 4:1 was associated with a 70\% decrease in mortality (de Lorgeril et al., 1994). A ratio of 2.5:1 reduced rectal cell proliferation in patients with colorectal cancer, and a ratio of 2:1 to 3:1 suppressed inflammation in patients with rheumatoid arthritis (Cleland et al., 2003). Newton (1996), reported an optimal ratio of 5:1 in humans, to prevent development of chronic diseases. These studies indicate that the optimal ratio may vary depending on the individual disease and the fact that chronic diseases are multigenic and multifactorial (Simopoulos, 2002). Consequently, it is quite possible that the optimal ratio in humans may vary with the disease under consideration. Although the optimal ratio of dietary n-6:n-3 fatty acids has not been determined in horses, data from other species indicate that dietary supplementation to increase the concentration of n-3 fatty acids could have beneficial effects for equine (King et al., 2008). Non-supplemented horses, are reported to maintain an average ratio of approximately 7.5:1 during the winter months when horses are primarily consuming grass hay and are reduced to approximately a 6:1 ratio during the spring when horses are consuming fresh green grass. King et al. (2008) reported horses receiving a marine based product formulated to provide $40 \mathrm{~g} / \mathrm{d}$ of EPA and DHA, sufficiently altered plasma concentrations to a ratio of 4.5:1. Based on human recommendations of a 5:1 
ratio, this study indicated that a minimum supplementation level of 30-35 g/d of EPA and DHA would be required to observe beneficial effects in horses.

For beneficial effects to occur, EPA and DHA must be present in circulation and incorporated into tissues. O'Connor et al. (2001) supplemented equine diets with fish oil containing $10.8 \%$ EPA and $8 \%$ DHA resulting in a 4.8 fold increase in plasma EPA and a 4.7 fold increase in plasma DHA following $63 \mathrm{~d}$ of supplementation. Furthermore, King et al. (2005) reported significant increases in plasma EPA and DHA after feeding an n-3 supplement for $28 \mathrm{~d}$. A more recent study found when horses were supplemented with $40 \mathrm{~g} / \mathrm{d}$ of EPA and DHA, peak EPA concentrations reached 13x control values, and peak DHA concentrations were approximately 10x control values (King et al., 2008). These results indicate significant increases in plasma concentrations of both EPA and DHA are highly correlated to the level of supplementation.

Cleland et al. (2003) determined plasma EPA levels of 3.2\% as an effective indicator of sufficient dietary n-3 PUFAs in humans by significantly suppressing the production of inflammatory cytokines and eicosanoids. Plasma EPA concentrations have an inverse relationship with the cytokines, tumor necrosis factor-alpha (TNF- $\alpha$ ) and interleukin-1 beta (IL-1 $\beta$ ), that are considered to be the central regulators of inflammation (Cleland, 2003). Caughey et al. (1997) observed that when EPA levels where $1.5 \%$ of the total fatty acids or greater, suppression of these inflammatory cytokines resulted. Furthermore, based on research completed in this laboratory, horses consuming $15 \mathrm{~g}$ of EPA and $20 \mathrm{~g}$ of DHA daily fulfilled this required $3.2 \%$ plasma 
concentration of EPA and DHA in order to elicit a response in suppressing the production of inflammatory mediators (Ross et al., 2007).

\section{Health Mitigation of Omega 3 Supplementation}

\section{Coronary Heart Disease}

The first epidemiological studies involving omega 3 fatty acids reported a lower occurrence rate of coronary heart disease among Greenland Inuit populations, which consume a traditional diet high in n-3 PUFAs (Dyenberg et al., 1975). Subsequent studies have further established a correlation between the fatty acid composition of the diet and the development of cardiovascular disease. These findings have led to the discovery of the potential health benefits associated with n-3 fatty acids (Dyenberg et al., 1975; Dyenberg et al., 1978).

Suggested mechanisms of n-3 fatty acids have focused on n-3 supplementation and eicosanoid metabolism, inflammation, and the production of cytokine growth factors. Das (2000) reported that n-3 fatty acids inhibit synthesis and release of proinflammatory cytokines, such as TNF- $\alpha$, IL-1 and IL-2, which are released during the early development of coronary heart disease. Dyenberg and Bang (1979) emphasized the importance of EPA in the prevention of heart attacks because of its antithrombotic effects, increased bleeding time, and reduced serum cholesterol concentrations.

Supplementation of EPA and DHA has also been shown to lower circulating triglyceride concentrations in both rats (Surette et al., 1992) and humans (Saynor and Gillott, 1992; Christenson et al., 1999). In horses, n-3 fatty acid supplementation decreased circulating levels of triglycerides at $63 \mathrm{~d}$ of supplementation (O'Connor et al., 
2007). The exact mechanism responsible for the decrease has not been determined. However, many studies suggest that n-3 fatty acids down-regulate enzymes associated with triglyceride synthesis (Marsh et al., 1987; Surette et al., 1992). Therefore, consistent data between animal models and randomized control trials have inferred an increase in n-3 fatty acids intakes from fish or supplements decreases the risk of fatal coronary heart disease (Kris-Etherton et al., 2002; He et al., 2004).

Exercise Tolerance

A high resting heart rate in humans is considered to be strongly linked to cardiovascular events, including mortality (Hjalmarson, 1990). Although the exact mechanism is not known, omega 3 fatty acids may have the potential to affect contractility of the heart. Leaf et al. (2003) found that n-3 fatty acids can inhibit the fast, voltage-dependent $\mathrm{Na}$ channels in cultured myocardiocytes. In horses, n-3 fatty acid supplementation lowered resting heart rates, and the authors suggested that an increased production of endothelium derived relaxing factor and/or a decrease in blood viscosity caused the effect (O’Connor et al., 2007).

Many researchers have hypothesized that n-3 fatty acid supplementation may provide additional benefits during exercise (Kris-Etherton et al., 2003; O'Connor and Lawrence, 2004; O’Keefe et al., 2006). In humans, omega 3 fatty acid supplementation resulted in lower maximum heart rate values and accelerated the return to resting heart rate following exercise (O'Keefe et al., 2006). Also, in both humans and horses undergoing treadmill exercise, the time to fatigue is directly related to the intensity of exercise, and that higher heart rates are associated with a faster time to fatigue (Hodgson 
et al., 1990). This study suggests lowering maximal heart rate and accelerating return to resting heart rate would be a great benefit to the equine athlete. Furthermore, clinical trials have demonstrated humans receiving n-3 supplementation had increased maximal oxygen consumption $\left(\mathrm{VO}_{2 \text { max }}\right)$ anaerobic threshold (Brilla and Landerholm, 1990). Therefore, these physiological changes due to $n-3$ supplementation may potentially improve exercise performance (Brilla and Landerholm, 1990).

\section{Joint Disease}

The development of joint disease involves the entire synovial joint, including the cartilage, synovium, and underlying bone. The cells within each of these tissues have independent capacities to initiate a response to injury, which can ultimately result in the degeneration of cartilage. The degeneration of cartilage in joint disease is characterized by 2 phases: a biosynthetic phase, in which attempts are made to repair the damaged extracellular matrix (ECM); and a degenerative phase, in which the activity of enzymes produced by chondrocytes digest the matrix, matrix synthesis is inhibited, and the consequent erosion of the cartilage is accelerated (Sandell and Aigner, 2001).

Data from experimental and clinical studies have provided evidence that $n-3$ fatty acids promote an anti-inflammatory response, making them potential therapeutic agents for inflammatory and autoimmune diseases, including rheumatoid arthritis (RA) in humans. The development of RA is characterized by flares of arthritis involving small and large joints and mediated by an exaggerated production of cytokines and eicosanoids (Zurier et al., 2003). The most consistent benefits of n-3 supplementation in individuals previously diagnosed with RA have been a reduction in morning stiffness 
and a decrease in joint tenderness (Cleland, 2003). In an earlier study, Cleland et al. (1988) reported tender joint score and grip strength improved in human patients receiving supplemental EPA and DHA. Similar results in other studies have shown n-3 PUFA supplementation improved pain outcome after 3 mo of supplementation, in particular patient assessed pain, which led to a reduction of non-steroidal antiinflammatory drugs (NSAIDs) usage (Goldberg and Katz, 2007). These studies indicated providing additional dietary n-3 fatty acids in the form of fish oil, which naturally contains high concentrations of EPA and DHA, reduced pain and other symptoms associated with patients diagnosed with RA.

It remains to be determined empirically whether the ability of EPA/DHA to reduce pain is due to one or more of the following possibilities: suppression of the inflammation underlying RA, or the direct effects on prostaglandin and cytokine production (Goldberg and Katz, 2007). Curtis et al. (2000) utilized bovine articular chondrocyte explants to demonstrate the ability of n-3 fatty acids to incorporate into chondrocyte membranes. This incorporation led to a reduction in expression and activity of proteoglycan degrading enzymes (aggrecanases) and expression of inflammationinducible cytokines (IL-1 and TNF- $\alpha$ ) produced through the COX pathway (Curtis et al., 2000). The effect of fish oil supplementation is most notably a reduction in TNF- $\alpha$ and IL-1. These pro-inflammatory cytokines are responsible for increased release of collagenases, stromelysin, and other enzymes associated with irreversible joint damage resulting in RA (Westacott and Shariff, 1996). These findings indicated that n-3 PUFAs, especially EPA and DHA, specifically affect regulatory mechanisms responsible for the 
progression of RA. Furthermore, previous studies have indicated that dietary fish oil supplementation can be utilized in the alleviation of several physiological parameters that cause and propagate arthritic disease (Curtis et al., 2000).

A reduction of pain and inflammation associated with RA resulting from n-3 PUFA supplementation led to investigations of animals diagnosed with OA (Munsterman et al., 2005). The osteoarthritic disease process involves the entire synovial joint, encompassing the synovium, cartilage and underlying bone. Supplemental EPA and DHA have shown promise in the therapeutic management of OA in canines, and could offer an alternative to prevent OA progression in other species. Initial in vitro studies in canine models investigated the biologic and molecular mechanisms involved in cartilage matrix degradation as the pathogenesis of degenerative joint disease progressed. Articular cartilage explants were exposed to a variety of cytokines, growth factors, and chemical mediators, which activated degradative enzymes and metabolic factors associated with cartilage degradation. Studies suggested when explants were exposed to EPA in particular, degradation decreased (Caterson et al., 2000; Curtis, 2002).

Based on previous in vitro studies, additional clinical studies were conducted utilizing n-3 supplementation as a nutritional aid for canines diagnosed with OA. Budsberg et al. (1999) reported consumption of a low $n-6$ to $n-3$ ratio (5.5:1) by healthy canines, prior to induction of $\mathrm{OA}$, was associated with less severe clinical and radiographic evidence of OA. Further studies in canines determined additional dietary EPA and DHA alleviated OA symptoms such as pain and swelling following $6 \mathrm{wk}$ of 
supplementation (Bierer and Bui, 2002). Canines receiving supplemental n-3 PUFAs had improved range of motion and ability to bear weight, as well as, a decrease in pain upon palpation of the affected joint. In a similar trial, force plate analysis revealed increased mean vertical peak force at $90 \mathrm{~d}$ of supplementation (Roudebush, 2008). These data from canine subjects reflects the potential of n-3 supplementation to effectively modulate inflammatory responses associated with OA.

\section{Impact of Equine Joint Disease}

Within the equine industry, lameness due to joint disease is considered to be the most prevalent cause of diminished athletic function and economic loss in performance horses (Goodrich and Nixon, 2006). Rossdale et al. (1985) reported that in Great Britain the greatest cause of attrition of young performance horses was lameness predominantly caused by joint disease. At Cornell University, approximately $20 \%$ of the horses admitted to the Large Animal Clinic from 1983 to 1987 were examined for lameness, and about $40 \%$ of these had joint disease as the primary problem (Todhunter and Lust, 1990). Therefore, the maintenance of normal joint function in order to prevent the onset of $\mathrm{OA}$ is a major concern to equine owners.

Traumatic OA, with associated inflammation of the synovial lining, is a common cause of lameness in young athletic horses (Munsterman et al., 2005). Once initial injury or degradation of cartilage occurs, inflammation results as an attempt to stop the damage. Initially, this process was designed to limit the use of the joint and promote healing, however, with continuous exercise and damage, excessive amounts of 
eicosanoids are released that result in chronic inflammation and pain, which elicit the degradation of articular cartilage (Palmer and Bertone, 1994).

Current treatments focus only on relieving pain and do not attempt to prevent further progression of the disease. Non steroidal anti-inflammatory drugs, such as phenylbutazone, are utilized for pain management only, and continuous administration can potentially lead to health problems, including gastric ulceration (MacAllister et al., 1993). More recently, interest has been placed on oral supplements containing glucosamine and/or chondroitin sulfate, however their efficacy has not been validated. Both compounds are required for the maintenance of healthy joints, and ideally consuming additional amounts of one or both of these compounds will provide an excess of resources required to maintain a healthy joint. Conte et al. (1991) reported the bioavailability of chondroitin sulfate in equines has been estimated at $13.2 \%$ of the amount ingested. This percentage then enters the circulatory system for incorporation into tissues, such as articular cartilage. However, even if the chondroitin sulfate is available in the plasma, both chondroitin sulfate and glucosamine can be filtered out of the blood after passage through the liver and which further decreases the usefulness in the body (Conte et al., 1991).

Prior work in horses diagnosed with OA has indicated the potential use of n-3 PUFAs as an alternative dietary supplement to current treatments. Woodward et al. (2005) reported a reduction in pain that corresponded to an increase in stride length in arthritic horses consuming $15 \mathrm{~g}$ of supplemental EPA and DHA on a daily basis. In a more recent study, $\mathrm{n}-3$ supplementation was effective in mitigating inflammation by 
lowering synovial WBC, and a tendency to decrease fibrinogen concentrations in horses receiving additional EPA and DHA (Manhart et al., 2009).

Previous studies have focused primarily on an arthritic model, with little information existing for the use of n-3 supplementation as a preventative measure against the development of OA. Benito et al. (2005) suggested inflammation was found to be more prominent in the early stages of OA when compared to the latter stages. This suggests the early stages of OA may respond favorably to supplementation of EPA and DHA.

\section{Bone and Cartilage Response to Exercise}

The use of horses for athletic competitions consists of conditioning and training regimens that are specific to individual performance events. Selection of training techniques for equine athletes is largely based upon subjective judgment and traditional training methods. Training involves increasing the work load (intensity and duration) and inducing a functional adaptation of subchondral bone and articular cartilage to the new demands (Smith et al., 1999). However, when young horses enter into training, their joints are subjected to stress that they may not be prepared to handle (Nielson et al., 1997). This can result in either a single overload or damage accumulation over a period of time that is insufficient to allow bone to properly adapt in response to increases in exercise load (Smith et al., 1999). The importance of this adaptation ensures that bone mass and cartilage architecture are appropriate for withstanding the mechanical loads placed upon joints (Nilsson and Westlin, 1971). 
Bone

Although bone is rather rigid, it responds to patterns of strain in the external environment. The ability of bone to alter its mass and shape according to the mechanical load placed upon it was first described in the nineteenth century by J. Wolff (Duncan and Turner, 1995). Wolff's law states the internal structure of a bone will change to accommodate new stresses which are placed on it as the function of bone is altered (Norwood, 1978). Osteocytes within bone detect variations in mechanical strain that fall outside the physiological window which then activates a series of processes that alter the ECM of bone tissue. This adaptation is necessary to return strain to a desired level and prevent structural failure (Norwood, 1978). When mechanical strain exceeds the upper physiological window, also referred to as minimum effective strain, bone undergoes changes to reduce strain to a level below the upper boundary (Duncan and Turner, 1995).

These changes in structure due to variations in mechanical load occur through the processes of bone modeling and remodeling. The majority of bone modeling occurs in the young animal, and it plays a major role in bone deposition in horses still experiencing skeletal growth. Modeling primarily alters the amount of bone present and serves to determine its form (Jee, 1988). In essence, modeling is the process by which the juvenile skeleton grows and develops into its adult form (Nielson et al., 1997). On the other hand, remodeling functions to replace immature (primary) bone with mature (secondary) bone in young growing animals, and allows for the replacement of both old and damaged bone throughout life (Nielson et al., 1997). An important distinction 
between bone modeling and remodeling is that the net amount of bone is either increased or decreased with bone modeling, while in remodeling, there tends to be no net change in the amount of bone present; remodeling simply replaces previously removed bone (Jee, 1988).

During remodeling, removal of old bone causes a temporary increase in the porosity of the bone that can decrease the ability of bone to resist stress, leaving the horse susceptible to micro fractures. According to Norwood (1978), re-absorption of old bone typically lasts for approximately $30 \mathrm{~d}$ and is then followed by the addition of new bone which lasts for approximately $1 \mathrm{wk}$. Nielson et al. (1997) reported long yearlings entering into race training had the highest incidence of bone related injuries during the period when bone density was the lowest (d 62 through d 120 of training). Therefore, it is important to consider an appropriate exercise program in order to achieve properly adapted bone in order to avoid fatigue failure when the bone is at its weakest.

\section{Articular Cartilage}

Special properties exhibited by articular cartilage are compressibility, elasticity, and self lubrication. These properties are due to the combined presence of collagen fibrils, densely concentrated proteoglycans, and glycoproteins (Neil et al., 2005). The cartilage that covers the articular surfaces of bones has two main functions: to limit stress applied to bone extremities and to provide a smooth surface necessary for joint function. Small numbers of chondrocytes are dispersed within the ECM that comprise articular cartilage. The principal role of this cartilage is to provide covering for the ends 
of bone at the diarthrodial joints (Dijkgraaf et al., 1995). Chondrocytes synthesize new matrix compounds which regulate degradation.

Mature articular cartilage is completely avascular and aneural without any lymphatic vessels or a limiting membrane (Neil et al., 2005). Since cartilage is aneural, pain perception in synovial joints is dependent on nerve endings in the synovium, capsule, muscles, and subchondral bone (Dijkgraaf et al., 1995). The uptake of nutrients and elimination of wastes are dependent upon diffusion established by a concentration gradient. Also, the viscoelastic structure of cartilage enables it to absorb energy from mechanical stresses. When stress is applied to cartilage, the tissue undergoes a slowly progressive deformation until the stress is removed and cartilage can return to its original shape (Dijkgraaf et al., 1995). Multiple proteins, hyaluronate, and collagen form a capsule to protect the chondrocyte from cell stresses.

Collagen is considered to be the monomeric unit of cartilage that contains a high amount of hydroxyproline and hydroxylysine amino acid residues in the shape of a triple helix. The anatomical arrangement of collagen fibrils (primarily type I and type II collagen) at various depths of cartilage provides structural support and counteracts swelling pressure of highly hydrophilic proteoglycans. This arrangement gives it tensile stiffness and strength (Goodrich and Nixon, 2006).

The ECM of articular cartilage determines biomechanical characteristics of the tissue, such as resilience and elasticity (Dijkgraaf et al., 1995). The ECM is continuously exposed to both anabolic and catabolic factors that vary depending on joint location. It is composed of water, type II collagen, proteoglycans, a smaller percentage 
of structural glycoproteins, and a small amount of lipids and inorganic compounds. Type II collagens are arranged as fibrils that are responsible for giving articular cartilage its tensile strength (Neil et al., 2005).

Proteoglycans prevent exposure of the exogenous and endogenous agents of articular cartilage to the rest of the body. A proteoglycan or a protein polysaccharide is the term commonly applied to a family of compounds whose basic structure is a single protein with chains of covalently attached glycosaminoglycans (GAGs). The largest and most abundant proteoglycan in cartilage is aggrecan, and it comprises 20 to $40 \%$ of the dry weight of articular cartilage (Neil et al., 2005). Proteoglycans are considered to a have a "comb like" structure with some regions of the protein assuming a tertiary structure. They are also known to be highly hydrophilic as they have a high waterbinding capacity, which counteracts with cartilage to constrain their full expansion and provides cartilage with its elasticity and shear strength (Dijkgraaf et al., 1995).

Proteoglycans are complex macromolecules that contain a core protein with branching GAGs that are covalently bound to a protein. The GAGs are long chain heteropolysaccharides composed of repeating disaccharide units. Precursors for GAGs include amino sugars (glucosamine) that are synthesized through the hexosamine synthetic pathway (Neil et al., 2005). The disaccharide consists of a non-nitrogenous sugar and a hexosamine that are linked by a glycosidic bond. The GAGs have repeating disaccharide units of hexosamine (glucosamine or galactosamine) alternating with another residue of glucuronate, iduronate, or galactose (Neil et al., 2005). The GAGs 
molecules are responsible for drawing in water molecules into the extracellular matrix and providing compressive stiffness (Goodrich and Nixon, 2006).

The primary GAG found within articular cartilage is chondroitin 6-sulphate. In smaller amounts, keratan sulphate, chondroitin 4-sulphate, and hyaluronic acid (HA) are found. Lubricin along with HA provides synovial fluid with its unique properties of boundary lubrication and steric hindrance that is important for reducing interaction of enzymes, antigens, or cytokines with target cells. Chondroitin 4 and 6-sulphate have identical repeating units, however, the sulphate ester group appears on carbon 4 of chondroitin 4-sulphate and on carbon 6 of chondroitin 6-sulphate (Dijkgraaf et al., 1995). Both chondroitin sulphates (4 and 6) are linked by a hydroxyl group of serine residues. Serine is bridged to the repeating units of the chondroitin sulphate by an oligosaccharide with a sugar sequence that resembles others within the chain (Goodrich and Nixon, 2006).

Similar to subchondral bone, the biochemical composition of articular cartilage is not homogenous, but rather, it adapts in response to biomechanical forces that are generated during early training and exercise that vary between different sites across the joint surface (Brama et al., 2000). As a result, each area has different inherent biomechanical and biochemical properties that are subjected to particular types of loads; including constant loads during weight bearing, intermittent loads during gait, and sudden loads during intense training or racing (Mcllwraith and Trotter, 1996). However, whereas bone is responsive to changes in exercise throughout life, remodeling of articular cartilage in considered a once-in-a-lifetime process, due to the extremely low 
turnover rate in mature individuals (van Weeren, 2008). Therefore, proper biomechanical loading is an important consideration for future strength and resistance of articular cartilage to injury (Brama et al., 2002).

In general, loading stimulates synthesis of extracellular matrix molecules, primarily proteoglycans, and enhances biomechanical stiffness of articular cartilage. However, this response is dependent upon both the magnitude and duration of load applied (McIlwraith and Trotter, 1996). Studies in canine stifle joints have demonstrated that moderate exercise $(4 \mathrm{~km} / \mathrm{d})$ stimulates proteoglycan synthesis; increases GAG concentration, and further increases the stiffness of the articular cartilage to indentation. In contrast, strenuous exercise $(20 \mathrm{~km} / \mathrm{d})$ decreases the GAG concentration within the extracellular matrix and does not increase the stiffness of articular cartilage (Jurvelin et al., 1986; O’Connor et al., 1988; Kiviranta et al., 1988). Similar to canines, in horses it has been hypothesized that regular joint loading during youth is important for the development of a well organized and strong articular cartilage collagen network (Brama et al., 2000 and Helminen et al., 2000). However, repeated trauma and stress to the joint through high impact loading may be associated with degenerative changes in the articular surface that may increase the risk of developing degenerative joint disease later in life (Billinghurst et al., 2003). Therefore, it appears there is a critical level after which the beneficial effect of exercise is overwhelmed by potential consequences of articular damage associated with loading. This is considered to be one of the most critical aspects in the training of young performance horses (McIlwraith and Trotter, 1996). 
When the reparative efforts of articular cartilage fail due to deficiencies in synthesis and assembly of the ECM, the breakdown of collagen fibrils can result. When type II collagen is cleaved (unwound) by collagenases, it forms tropocollagen (composed of 3 identical $\alpha$ helixes) that contains an amino and a carboxy terminus.

Tropocollagen is then further degraded by collagenases to produce $\alpha$ chains and peptide fragments that expose normal hidden epitopes (McIlwraith and Trotter, 1996). These small peptide fragments of cartilage molecules are either taken up by the chondrocytes and enter into circulation or are further degraded by lysosomal enzymes or lost into synovial fluid by diffusion (Figure 3; Lohmander et al., 1994).

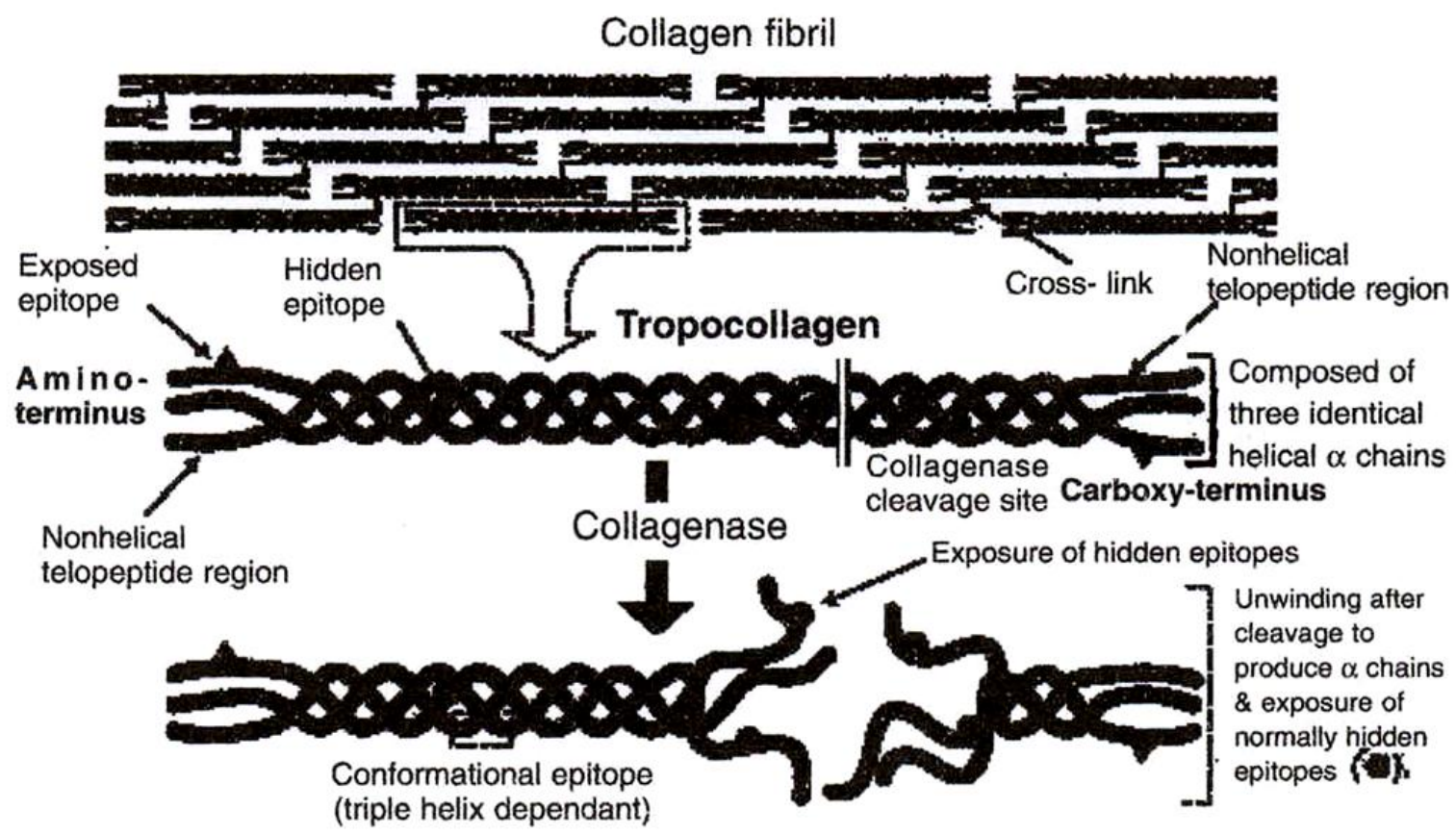

Figure 3. Structure of type II collagen (Poole, 1992). 


\section{Anabolic Markers Relative to Joint Health}

All components of articulating joints participate in load transmission, and failure of bone and articular cartilage of a joint may lead to exercise-induced damage (Billinghurst et al., 2003). The ability to detect these changes at an early stage would potentially enhance the ability to modulate exercise protocols in order to avoid irreversible damage to joint structures. One potential diagnostic tool, which has been receiving attention for the early diagnosis of $\mathrm{OA}$, is the evaluation of body fluid levels of by-products of articular cartilage metabolism (Billinghurst et al., 2003).

C-propeptide of type II collagen (CPII) represents a marker of type II collagen synthesis (Billinghurst et al., 2003). The CPII molecule is proteolytically cleaved from the procollagen strand during fibril formation, and the concentration of this peptide has been directly related to the rate of collagen synthesis, which has been shown to increase in arthritic joints. In human clinical trials, CPII concentrations are higher in articular cartilage, serum, and synovial fluid from patients with joint disease (Rizkalla et al., 1992; Shinmei et al., 1992; Nelson et al., 1994). Frisbie et al. (1999) reported CPII concentrations increase ( $23.7 \pm 1.89$ to $28.6 \pm 1.84)$ in response to osteochondral fragmentation in horses.

Articular cartilage is composed of an ECM that contains primarily type II collagen and a large aggregating proteoglycan known as aggrecan. Aggrecan molecules are responsible for the compressive strength of joint tissue (Billinghurst et al., 2003). Previous studies have indicated that aggrecan synthesis is reflected by the presence of the epitope 846 (CS-846), which is found on newly synthesized chondroitin sulfate 
chains of cartilage proteoglycan molecules (Rizkalla et al., 1992; Poole et al., 1994).

The CS-846 is restricted to the largest aggrecan molecules in normal articular cartilage, and these aggrecan molecules exhibit complete aggregation with hyaluronan, unlike the majority of other aggrecan molecules (McIlwraith and Trotter, 1996). In humans, concentrations of CS-846 were approximately three-fold greater in injured joints $(0.78$ $\mu \mathrm{g} / \mathrm{mL})$ when compared with normal, healthy joints $(0.26 \mu \mathrm{g} / \mathrm{mL}$; Lohmander et al., 1994).

The concentration of this epitope is highest in fetal tissue and progressively declines with skeletal development, but increases in diseased articular cartilage. In normal adult humans, serum concentrations of CS-846 were very low (Glant et al., 1986). However, in patients with OA, when synthesis is increased, concentrations of this epitope were elevated in both articular cartilage and synovial fluid (Rizkalla et al., 1992; Lohmander et al., 1994). In horses, similar to CPII, increased serum concentrations of CS-846 ranged from $0.06 \pm 0.09 \mu \mathrm{g} / \mathrm{mL}$ in non-affected joints to 0.26 $\pm 0.05 \mu \mathrm{g} / \mathrm{mL}$ in affected joints and have been associated with osteochondral fragmentation suggesting increased synthesis of ECM components (Frisbie et al., 1999).

A recent study was conducted to discriminate between changes in biomarkers associated with exercise in horses compared to those in horses with induced OA via an osteochondral fragment (Frisbie et al., 2008). Synovial fluid concentrations of CPII and CS-846 were elevated in horses with induced OA, and remained higher throughout the study when compared to horses receiving exercise alone. Following the onset of exercise (simulated strenuous exercise of race training), serum concentrations of CPII 
and CS-846 increased in normal joints. Further, there were correlations between concentrations of biomarkers found in synovial fluid compared to serum. Synovial fluid concentrations of CPII and CS-846 were 50\% and three-fold higher, respectively, in synovial fluid compared to serum of the same horses (Frisbie et al., 2008). This correlation is particularly useful in screening clinical cases for OA when horses are in active training. Routine synovial fluid acquisition is difficult to obtain in elite equine athletes, however serum sampling is well tolerated (Frisbie et al., 2008).

The early stages of OA are difficult to diagnose. Joint structure and function are often altered substantially before seeking veterinary care. The progression of OA requires an early diagnosis that can allow for treatment that may prevent further cartilage destruction and joint failure. Diagnostic tests that can detect and monitor molecular changes early in the pathogenesis of OA would be potentially useful (Frisbie et al., 2008).

\section{Inflammatory Markers}

It has also been suggested that levels of inflammatory mediators in synovial fluid could potentially be used as markers of OA. Although blood samples are considered to be easier to obtain, concentrations of markers within synovial fluid have been found to be more indicative of joint disease, as many markers may be eliminated by the time they enter into circulation (Frisbie et al., 2008).

Synovial WBC is commonly utilized to identify joint problems, with the normal equine concentration being reported to range from 67 to 188 cells/mL (Van Pelt, 1974; Persson, 1971). Bertone et al. (2001) reported correlations between WBC and the 
inflammatory markers TNF- $\alpha$, IL-1, and IL-6. The interactions of these 3 main cytokines are integrally involved in synovial metabolism and arthritis, and are commonly associated with the stimulation of articular cartilage matrix degradation (May et al., 1992). Concentrations of IL-6 are considered to be the most sensitive and more specifically related to joint disease. Concentrations of IL-6 increase in the early stages of OA. Therefore, the use of WBC can be considered a useful indicator of joint disease (Bertone et al., 2001).

Other inflammatory markers of joint disease include TP and SG of synovial fluid. Van Pelt (1974) determined values of TP as $1.81 \pm 0.26 \mathrm{~g} / \mathrm{dL}$ and $\mathrm{SG}$ of $1.013 \pm 0.002$ to be considered normal. The synovial fluid TP concentration is approximately 25 to $35 \%$ of the plasma protein concentration of the animal (McIlwraith and Trotter, 1996). The TP concentration increases with synovitis and with increasing inflammation (McIlwraith and Trotter, 1996). When total protein concentrations become greater than $2.5 \mathrm{~g} / \mathrm{dl}$, it is appropriate to consider the fluid abnormal and concentrations $\geq 4 \mathrm{~g} / \mathrm{dL}$ indicates severe inflammation. The TP concentration and WBC are classic indicators of inflammation and increased vascularity but, TP concentration is considered to be a more sensitive indicator to alterations within joints affected with OA (Frisbie et al., 1999). Similar to TP, the SG of synovial fluid increases with arthritis (Rohde et al., 1997). Persson (1971) determined TP and SG concentrations also increased with increasing intensity of exercise. Therefore, concentrations of these markers could potentially be utilized to reflect the progression of $\mathrm{OA}$ and the response of articular joints to exercise. 


\section{Conclusion}

In summary, early training and exercise is a time of adaptation of bone and soft tissue. When young horses enter into training their joints often undergo repeated trauma and stress, which can potentially lead to the production of inflammatory mediators including both cytokines and eicosanoids. Over time, this leads to the enzymatic breakdown of articular cartilage through the release of collagenases. These collagenases release small peptide fragments (including CPII and CS-846) that are released into circulation.

It is evident that joint damage that occurs early in a horse's career often can influence the development of OA later in life. One preventative strategy is the inclusion of n-3 fatty acids in the diet. One compounding effect, however, is the dietary sources of fat added diets are high in n-6 fatty acids and could potentially be increasing the inflammatory potential. Evidence suggests that EPA in particular has the ability to mitigate inflammation through the COX pathway. Previous literature has indicated the potential for n-3 fatty acids to mitigate inflammation related to OA (Calder, 2002;

Manhart et al., 2009). However, these studies have utilized an arthritic model with little information existing relative to a prevention strategy.

Measuring the effectiveness of n-3 supplementation poses great difficulty. As performance characteristics are often difficult to measure objectively, with other options including arthrocentesis is not practical for industry use. Due to these difficulties, the potential use of anabolic markers, including CPII and CS-846, can be utilized as noninvasive means to evaluate joint health. Therefore based on previous literature, these 
anabolic markers could potentially be utilized for the early detection of OA and as a means to evaluate the efficacy of $\mathrm{n}-3$ supplementation in young performance horses. 


\section{CHAPTER III}

MATERIALS AND METHODS

\section{Horses and Dietary Treatments}

Sixteen Quarter horses (mares and geldings) from the Texas A\&M University Horse Center herd were utilized in a randomized complete block design for a $140 \mathrm{~d}$ trial. Horses ranged from 2 to $4 \mathrm{yr}$ and weighed 357 to $439 \mathrm{~kg}$. Horses were initially blocked by BW, age, and sex. All procedures and handling of animals was approved by the Institutional Animal Care and Use Committee (AUP\# 2007-20).

Horses were randomly assigned within block to one of two dietary treatments. Dietary treatments were formulated to be isocaloric and isonitrogenous with a control diet $(n=8)$ that consisted of a $12 \%$ crude protein $(C P)$ pelleted concentrate (Producer's Cooperative Association, Bryan, TX) offered at 1\% BW (as fed) per d. The treatment diet $(n=8)$ consisted of the pelleted concentrate fed at $0.75 \% \mathrm{BW}$ (as fed) with an additional $350 \mathrm{~g}$ per $\mathrm{d}$ of a commercial marine-based omega 3 supplement (JBS United Feeds, Inc., Sheridan, IN) that was stored in individual containers (237 mL plastic storage container, Thermo Fisher Sci., Waltham, MA) and mixed with concentrate immediately prior to feeding. The amount of supplement provided was based on previous studies (Ross et al., 2007; Manhart et al., 2009) to provide $15 \mathrm{~g}$ EPA and $20 \mathrm{~g}$ of DHA. All horses received approximately 1\% BW (as fed) Coastal Bermudagrass hay (Cynodon dactylon) per d with ad libitum access to water. 
All horses were housed individually in 3 x 3 m stalls and fed their respective dietary treatments individually at $12 \mathrm{hr}$ intervals, with intakes and refusals measured daily. Throughout the trial, horses consumed an average of $3.9 \mathrm{~kg}$ of concentrate and approximately $8.0 \mathrm{~kg}$ of hay per $\mathrm{d}$. Bodyweight was determined and recorded biweekly and concentrate intake adjusted accordingly. Body condition score (BCS) was also determined biweekly using the 1 to 9 scale described by Henneke et al. (1983). Grain, hay, and supplement were sampled throughout the trial and analyzed for nutrient content, including gross energy (Table 1) and fatty acid concentrations (Table 2).

Table 1. Gross energy content (Mcal/kg) of diet components

\begin{tabular}{lc}
\hline \hline Component & Gross Energy \\
\hline Concentrate $^{\mathrm{a}}$ & 3.85 \\
Coastal Bermudagrass Hay & 3.97 \\
Supplement $^{\mathrm{b}}$ & 5.10 \\
\hline${ }^{\mathrm{a}} 12 \%$ CP pelleted concentration (Producer's Cooperative Association, Bryan, TX) \\
${ }^{\mathrm{b}}$ Commercial marine based n-3 product (JBS United Feeds, Inc., Sheridan, IN)
\end{tabular}


Table 2. Unsaturated fatty acid profile of concentrate, coastal bermudagrass hay, and marine based n-3 supplement (\% of total dietary fatty acids)

\begin{tabular}{lccc}
\hline Fatty Acid & Concentrate $^{\mathrm{a}}$ & Coastal Bermudagrass Hay & Supplement $^{\mathrm{b}}$ \\
\hline C 16:1 (PA) & 0.00 & 1.72 & 0.04 \\
C 18:1 (OA) & 0.67 & 1.82 & 0.08 \\
C 18:2n6 (LA) & 1.61 & 0.85 & 0.15 \\
C 18:3n3 (ALA) & 0.14 & 0.17 & 0.32 \\
C 20:1 (ESA) & 0.02 & 0.19 & 0.00 \\
C 20:4n6 (AA) & 0.00 & 0.20 & 0.00 \\
C 20:3n6 (DGLA) & 0.00 & 0.03 & 0.00 \\
C 20:5n3 (EPA) & 0.00 & 2.18 & 0.05 \\
C 22:6n3 (DHA) & 0.00 & 2.47 & 0.05 \\
n-6:n-3 ratio & $12: 1$ & $1: 5$ & $1: 3$ \\
\hline a 12\% CP pelleted concentration (Producer's Cooperative Association, Bryan, TX) \\
b Commercial marine based n-3 product (JBS United Feeds, Inc., Sheridan, IN)
\end{tabular}

\section{Exercise Protocol}

Phase I: Ground Work and Early Training

Exercise was conducted in conjunction with an equine behavior and training class at Texas A\&M University. All horses were randomly assigned to students in the class $(n=13)$ or to graduate students $(n=3)$ affiliated with the project. Horses were exercised $5 \mathrm{~d} / \mathrm{wk}$ for of 30 to $40 \mathrm{~min}$ per $\mathrm{d}$ in accordance with the course requirements.

During the first $9 \mathrm{wk}$ of the trial, exercise activities consisted of longing at 10 to 20 min intervals with only initial saddling and mounting for 10 to 20 min per session. 
Activities under saddle during this time consisted of walking and steady jogging. As the semester progressed, longing time decreased (10 to 15 min per d) and more emphasis was placed on increasing duration and intensity of riding (20 to $25 \mathrm{~min}$ per d). Riding throughout the semester was conducted as a group, which consisted primarily of walking, trotting, and loping circles in both directions, as well as, straight line work. Horses were asked to perform hindquarter/forehand pivots, along with side passing. Phase II: Advanced Maneuvers and Moderate Workload

At the completion of the class (wk 14), a group of riders $(n=4)$ were randomly assigned to a daily rotation of horses to ensure that all horses were ridden by all riders each wk. Intensity and duration of the exercise was increased for the final $30 \mathrm{~d}$ of the trial to simulate the increased workload that accompanies early training of the young horse. Workouts consisted primarily of riding (30 min) with only light longing (10 min). Additional time was devoted to more difficult maneuvers including two-tracking, simple lead changes, counter cantering, rollbacks, and pivoting.

Type of activity, duration (min), along with direction was monitored daily in order to maintain consistent workloads across all horses throughout the trial. Heart rate monitors (Equ Pulse Co., San Jose, CA) were utilized on a daily basis beginning at wk 10, when all horses were under saddle, in order to quantify workload on randomly selected horses (Table 3). A daily log was maintained to record exercise load and any incidence of injury or lameness. 
Table 3. Heart rates expressed in beats per minute (bpm) of randomly selected horses in response to exercise

\begin{tabular}{ccccccc}
\hline & \multicolumn{2}{c}{ Activity } & \multicolumn{4}{c}{ Heart Rate (bpm) } \\
\cline { 2 - 7 } Week $^{1}$ & $\begin{array}{c}\text { Longed } \\
(\mathrm{min})\end{array}$ & $\begin{array}{c}\text { Ridden } \\
(\mathrm{min})\end{array}$ & Minimum & Maximum & Mean & Median \\
\hline $1-9$ & $10-20$ & $10-20$ & ---- & --- & ---- & --- \\
10 & $11-15$ & $20-25$ & 55 & 155 & 115 & 119 \\
11 & $10-15$ & $20-25$ & 42 & 189 & 94 & 95 \\
12 & $10-15$ & $20-25$ & 42 & 190 & 101 & 93 \\
13 & $10-15$ & $20-25$ & 49 & 207 & 114 & 110 \\
14 & $10-15$ & $20-25$ & 51 & 212 & 130 & 119 \\
15 & $10-15$ & $25-30$ & 34 & 201 & 115 & 110 \\
16 & $10-15$ & $25-30$ & 30 & 217 & 103 & 100 \\
17 & $10-15$ & $25-30$ & 33 & 187 & 93 & 88 \\
18 & $10-15$ & $25-30$ & 36 & 189 & 100 & 99 \\
19 & $10-15$ & $25-30$ & 50 & 170 & 109 & 108 \\
20 & $10-15$ & $25-30$ & 34 & 200 & 107 & 105 \\
\hline
\end{tabular}

\footnotetext{
${ }^{1}$ Weeks 1-9 primarily consisted of longing and introduction to saddle; wk 10 all horses were under saddle. Beginning in wk 13 difficulty of maneuvers was increased (lope departures, loping circles, hindquarter pivots). After wk 14 the remaining exercise was completed by small group of rider $(\mathrm{n}=4)$; increased difficulty of maneuvers (rollbacks, spins, stops) and workload intensity (time devoted at the extended trot and lope).
}

\section{Sample Collection}

Synovial fluid and serum samples were obtained every $28 \mathrm{~d}$ throughout the $140 \mathrm{~d}$ trial. Sampling occurred on Fridays immediately following the completion of class. This allowed horses $2 \mathrm{~d}$ to recover before exercise began again on the following Monday. Blood samples were obtained via jugular venipuncture. Serum samples intended for CS-846 and CPII analysis were collected in non-additive sterile blood collection tubes (Kendall Co., Mansfield, MA) and remained at room temperature for approximately $1 \mathrm{hr}$ prior to centrifugation. Samples were centrifuged at $2700 \mathrm{x} g$ at 
$10^{\circ} \mathrm{C}$ for $20 \mathrm{~min}$ (ALC, PM140R, Thermo Fisher Sci., Waltham, MA), and then harvested and stored at $-20^{\circ} \mathrm{C}$ for later analysis.

Carpal arthrocentesis was performed by veterinarians from the Texas A\&M University Large Animal Clinic (College Station, TX). Horses were sedated using Xylazine $\mathrm{HCl}$ (RX Veterinary Products, Westlake, TX) administered intravenously at the recommended dose of $0.5 \mathrm{mg} / \mathrm{kg}$ of $\mathrm{BW}$. The joint was aseptically prepped using a alternating applications of Betadine scrub (Providine-iodine, 5\%, Purdue Frederick Company, Stamford, CT) and 70\% alcohol (Isopropyl Alcohol 70\%, Aaron Industries, Lynwood, CA) immediately prior to arthrocentesis. For sampling consistency, ease of collection, and to obtain the volume of fluid required, the right radial carpal joint was aseptically aspirated by use of a 20-gauge x 1in needle (Kendall Co., Mansfield, MA) inserted medial to the extensor carpi radialis tendon in the palpable depression between the radial carpal bone and the third carpal bone, to a depth of approximately 0.5 in to avoid unnecessary contact with articular cartilage (McIlwraith and Trotter, 1996). Synovial fluid was extracted (1 to $4 \mathrm{~mL}$ ) into a $6 \mathrm{~mL}$ syringe (Kendall Co.) and then transferred to tubes containing EDTA. Samples were then divided into small aliquots and stored in microcentrifuge tubes (VWR, West Chester, PA) at $-20^{\circ} \mathrm{C}$ for later analysis.

\section{Sample Analysis}

\section{Gross Energy Determination}

Gross energy was determined for grain, hay and supplement samples. Samples were not dried prior to analysis. All samples were ground through a $1 \mathrm{~mm}$ mesh screen 
(Thomas Model 4 Wiley Mill, Thomas Scientific, Swedesboro, NJ). Gross energy values were determined from a $1.0 \mathrm{~g}$ composite sample utilizing a bomb calorimeter (Par 6300 oxygen-bomb calorimeter, Parr Instrument Co., Moline, IL).

Synovial Fluid and Serum Analysis

One aliquot of synovial fluid was immediately transported to the Texas Veterinary Medical Diagnostic Lab (College Station, TX) following arthrocentesis in order to determine WBC, TP, and SG utilizing a Celdyne 3700 Cell Counter (Abbott Industries, Abbott Park, IL).

A commercially available ELISA kit (IBEX Pharmaceuticals Inc., Quebec, Montreal, Canada) was utilized to evaluate CPII concentrations in serum samples. The competitive immunoassay measured type II collagen carboxy propeptides. Briefly, standards and samples were first diluted (1:2) with an assay buffer solution provided by the manufacturer. Samples were then added to a polypropylene mixing plate, followed by a rabbit polyclonal antibody specific for the CPII peptide. Following the preincubation period, $80 \mu \mathrm{L}$ from each well was transferred from the mixing plate to the corresponding well of the ELISA plate. The plate was then allowed to incubate $2 \mathrm{hr}$ at 20 to $25^{\circ} \mathrm{C}$ on the plate shaker at $650 \mathrm{rpm}$ to allow the antibody to bind. The plate was then washed (Labsystems Wellwash 4, Thermo Fisher Sci., Waltham, MA) 6 times and blot dried after the final wash. After washing, goat anti-rabbit horseradish peroxidase (GAR-HRP) conjugate was added. The plate was covered and incubated for $30 \mathrm{~min}$ at 20 to $25^{\circ} \mathrm{C}$ at $650 \mathrm{rpm}$. The ELISA plate was again washed 6 times as previously described and blot dried after the last wash. Tetra-methylbenzidine (TMB) buffer 
warmed at $25^{\circ} \mathrm{C}$ was added to each well and all samples were incubated again on a plate shaker at $650 \mathrm{rpm}$ and were monitored for color development. The HRP degrades the peroxide and oxidizes TMB to form a blue product. At the completion of the incubation period, the reaction was stopped and the signal amplified by adding $100 \mu \mathrm{L}$ of stop solution/well, which converted the product from a blue to yellow color. Samples were quantified by utilizing a plate reader (BioRad 680 Microplate Reader, BioRad Laboratories, Hercules, CA) at an optical density (OD) of $450 \mathrm{~nm}$. The optical density is inversely proportional to the amount of CPII that is present in the samples.

Serum concentrations of CS-846 were determined utilizing a commercially available ELISA kit (IBEX Pharmaceuticals). This assay measured an epitope of chondroitin sulfate chains of the cartilage proteoglycan aggrecan. Briefly, standards and unknown were diluted 1:5 (10 $\mu \mathrm{L}$ serum $+40 \mu \mathrm{L}$ Buffer III) with a buffer solution that was provided by the manufacturer and added to the appropriate wells of the goat antimouse antibody coated microtitre plate. Next, the CS-846-Biotin was added to all the wells. Diluted mouse CS-846 antibody (50 $\mu \mathrm{L} /$ well) was then added to the ELISA plate to start the competition. The plate containing the mixture was incubated on a high speed titre plate shaker (Shaker ORBIT P4 No. 687) at $650 \mathrm{rpm}$ for $2 \mathrm{hr}$ at room temperature $\left(25^{\circ} \mathrm{C}\right)$. Following the incubation, the ELISA plate was washed 6 times using a Labsystems Wellwash 4, as described above, and blotted dry on the final wash. Diluted Streptavidin-HRP conjugate was added (100 $\mu \mathrm{L} /$ well) to each well in order to bind any bound CS-846-Biotin. The plate was then washed again incubated on the plate shaker at $650 \mathrm{rpm}$ for $30 \mathrm{~min}$ at $25^{\circ} \mathrm{C}$. The plate was washed again 6 times and blotted after the 
final wash as described previously and $100 \mu \mathrm{L} /$ well of TMB was added to each well placed on the shaker at $650 \mathrm{rpm}$ for $30 \mathrm{~min}$ at $25^{\circ} \mathrm{C}$ while color change was monitored. Stop solution was then added (100 $\mu \mathrm{L} /$ well) to stop the reaction and amplify the signal with an acid which converts the product from a blue to yellow color and the plate was read (BioRad 680 Microplate Reader) at $450 \mathrm{~nm}$ within $10 \mathrm{~min}$. The optical density recorded is inversely proportional to the amount of the epitope.

\section{Statistical Analysis}

All data were analyzed as a randomized complete block design using the PROC MIXED procedure of SAS (SAS Inst., Inc., Cary, NC). The model contained effects for treatment, day, and treatment $x$ day interaction. Main effects were considered significant when $P<0.05$ and a trend toward significance when $P<0.10$. 


\section{CHAPTER IV}

\section{RESULTS AND DISCUSSION}

\section{Body Weight and Body Condition Score}

There was no influence of dietary treatment on BW (Figure 4) or BCS (Figure 5) $(\mathrm{P}=0.49$ and $\mathrm{P}=0.28$, respectively). However, there was an influence of time on both BW and BCS $(\mathrm{P}<0.01)$. All horses, regardless of treatment, gained BW and BCS over the $140 \mathrm{~d}$ trial. It is important to note that dietary treatments exceeded the recommended DE requirement of young horses performing moderate exercise (21.46 Mcal/d; NRC, 2007) with treatment horses receiving approximately $34.80 \mathrm{Mcal} / \mathrm{d}$ and control horses receiving $34.10 \mathrm{Mcal} / \mathrm{d}$.

Furthermore, there was a treatment $\mathrm{x} d$ interaction $(\mathrm{P}<0.05)$, with treated horses having a higher BCS on d 140 when compared to control horses. This could be attributed to the lower heat increment associated with fat supplemented diets, especially when the ambient temperature was high. Ambient temperature ranged from $9^{\circ} \mathrm{C}$ on $\mathrm{d} 0$ of the trial in January, 2009 and averaged $35^{\circ} \mathrm{C}$ when approaching d 140 of the trial. This peak in ambient temperature also corresponded to phase II of the trial when intensity and duration of exercise increased. Other researchers in similar climactic conditions have noted an increased efficiency of gain when young, exercising horses were fed fat supplemented diets, and they noted that fat supplemented horses had lower total heat production (Scott et al., 1992) and lower energy requirements for maintenance (Potter et al., 1990). This could be due to the replacement of fermentable carbohydrates 
with fat in the concentrate portion of the diet, which lowers the thermal load placed upon an exercising horse. Therefore, relative to this study, treated horses may have been able to maintain body composition more readily, resulting in a higher BCS when compared to control horses, particularly when ambient temperature increased.

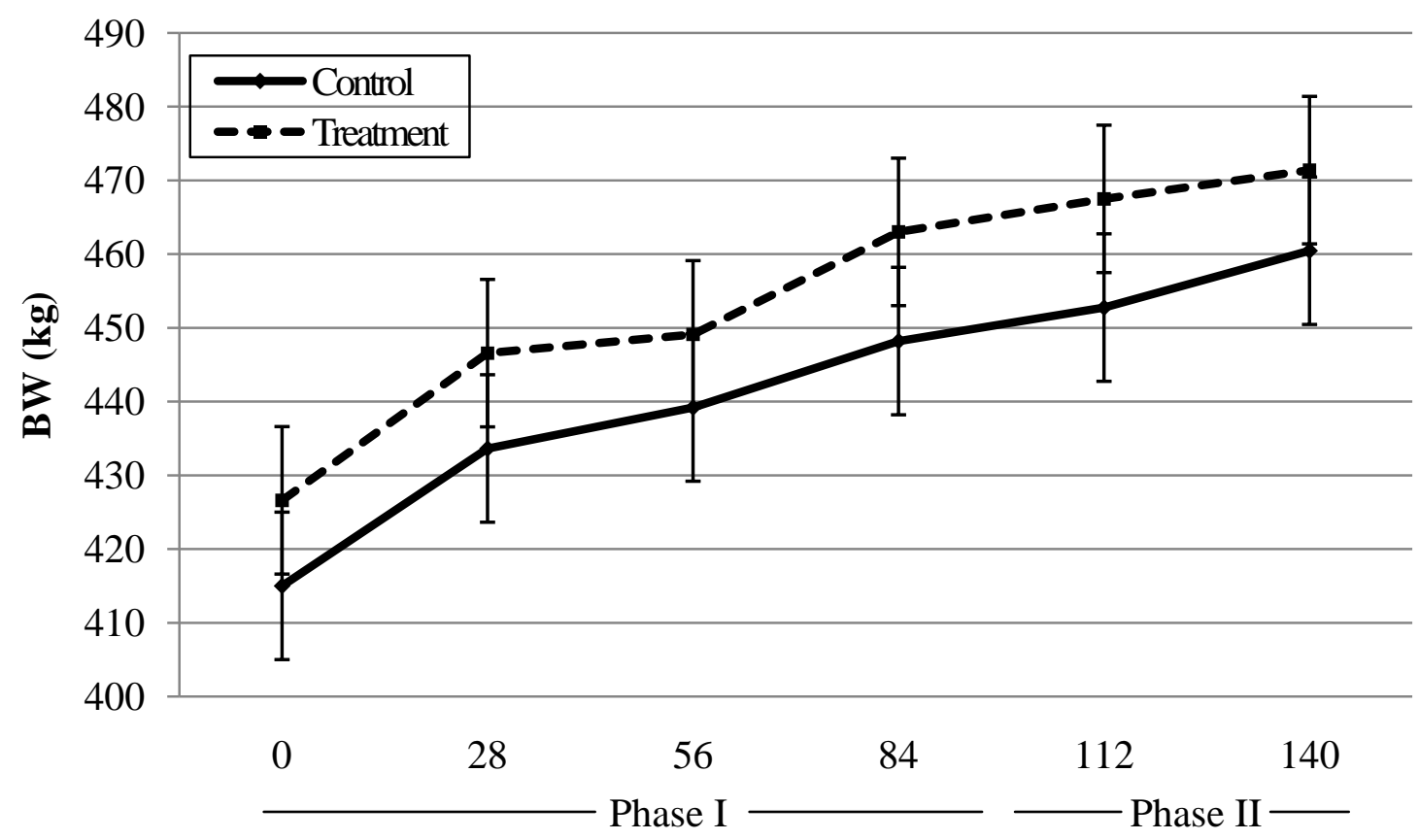

Time (d)

Figure 4. Changes in BW (kg) over time (d) beginning with phase I (d 0 to 110) consisting of ground work and early training to phase II (d 111-140) representing moderate workload and application of advanced maneuvers in horses receiving a pelleted concentrate with (Treatment) or without (Control) supplementation of a marine based omega 3 product. 


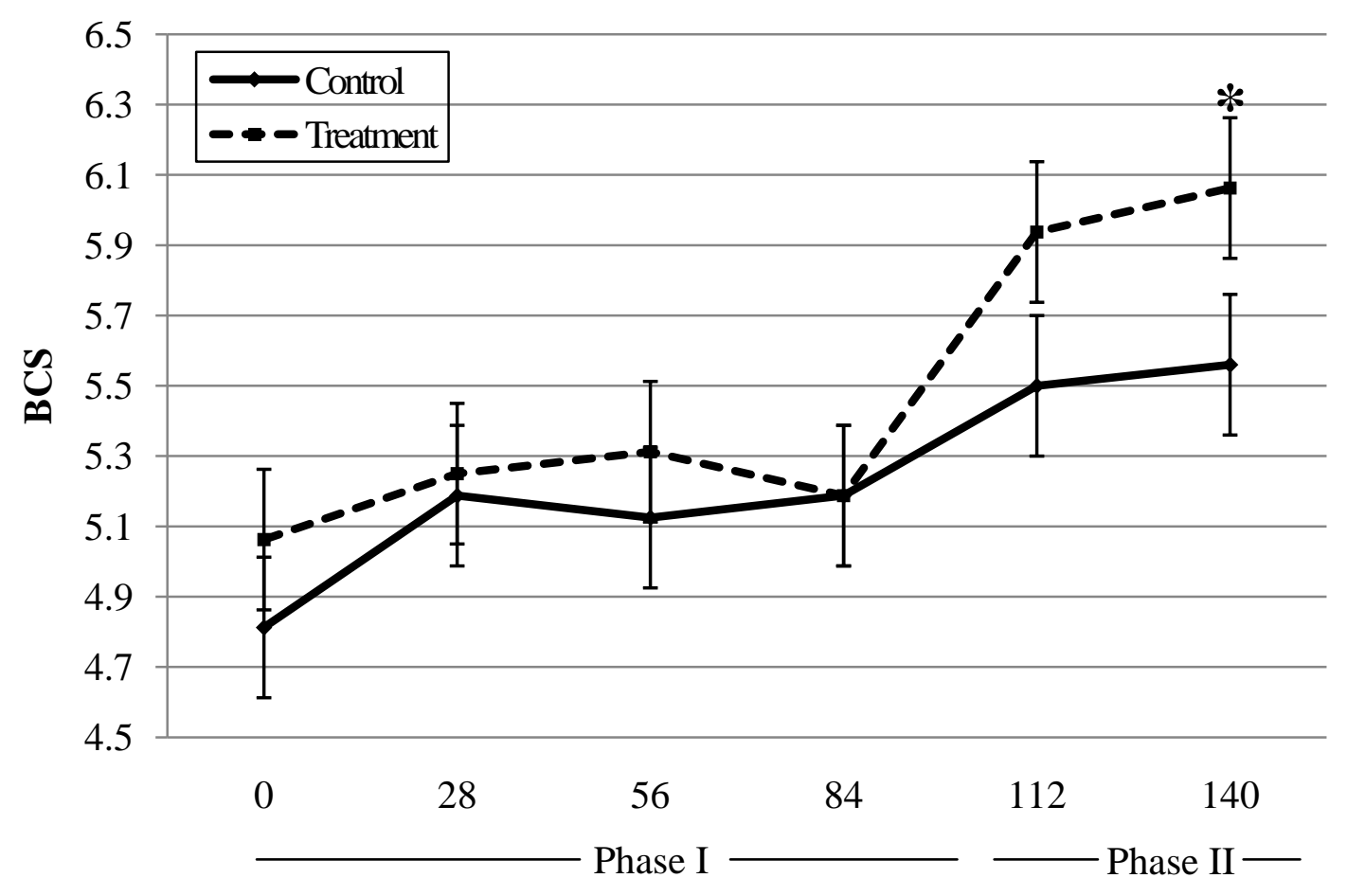

Time (d)

Figure 5. Changes in body condition score (BCS) over time (d) beginning with phase I (d 0 to 110) consisting of ground work and early training to phase II (d 111-140) representing moderate workload and application of advanced maneuvers in horses receiving a pelleted concentrate with (Treatment) or without (Control) supplementation of a marine based omega 3 product. ${ }^{*}$ Denotes treatment $\mathrm{x}$ time interaction, $\mathrm{P}<0.05$.

\section{Serum Anabolic Markers}

Dietary treatment did not influence serum concentrations of CPII or CS-846 (P = $0.47, \mathrm{P}=0.49$, respectively). Concentrations of CPII increased over time $(\mathrm{P}<0.01$; Figure 6) while concentrations of CS-846 tended to increase over the $140 \mathrm{~d}$ trial $(\mathrm{P}=$ 0.09; Figure 7) in response to increasing exercise load from phase I to phase II. The greatest increase in serum CPII concentrations was observed during the final $30 \mathrm{~d}$ of the 
trial which corresponds to Phase II of the experiment when exercise intensity increased, while CS-846 concentrations tended to gradually increase over time.

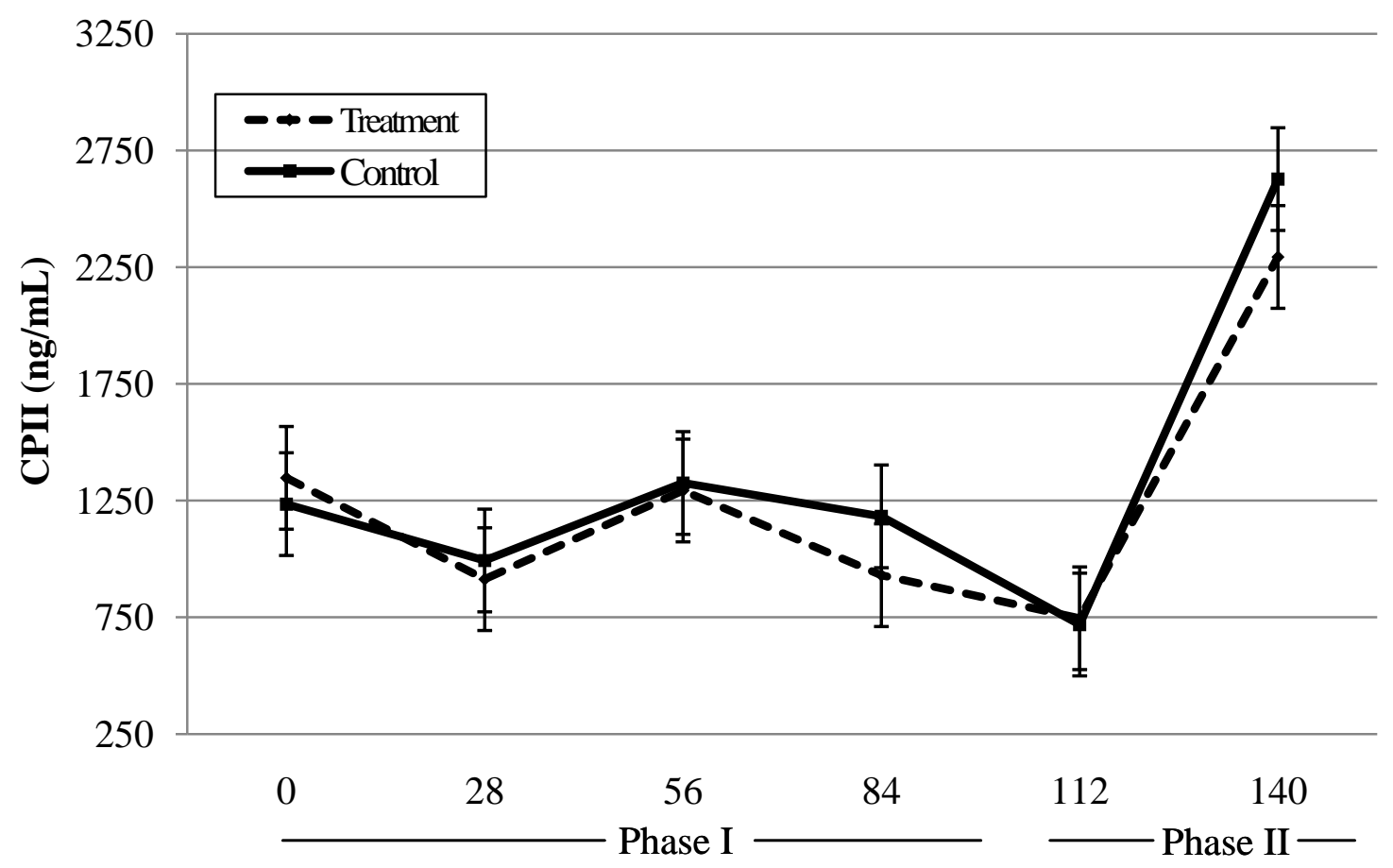

Time (d)

Figure 6. Serum concentrations $(\mathrm{ng} / \mathrm{mL})$ of the carboxypeptide of type II collagen (CPII) over time (d) beginning with phase I (d 0 to 110) consisting of ground work and early training to phase II (d 111-140) representing moderate workload and application of advanced maneuvers in horses receiving a pelleted concentrate with (Treatment) or without (Control) supplementation of a marine based omega 3 product. 


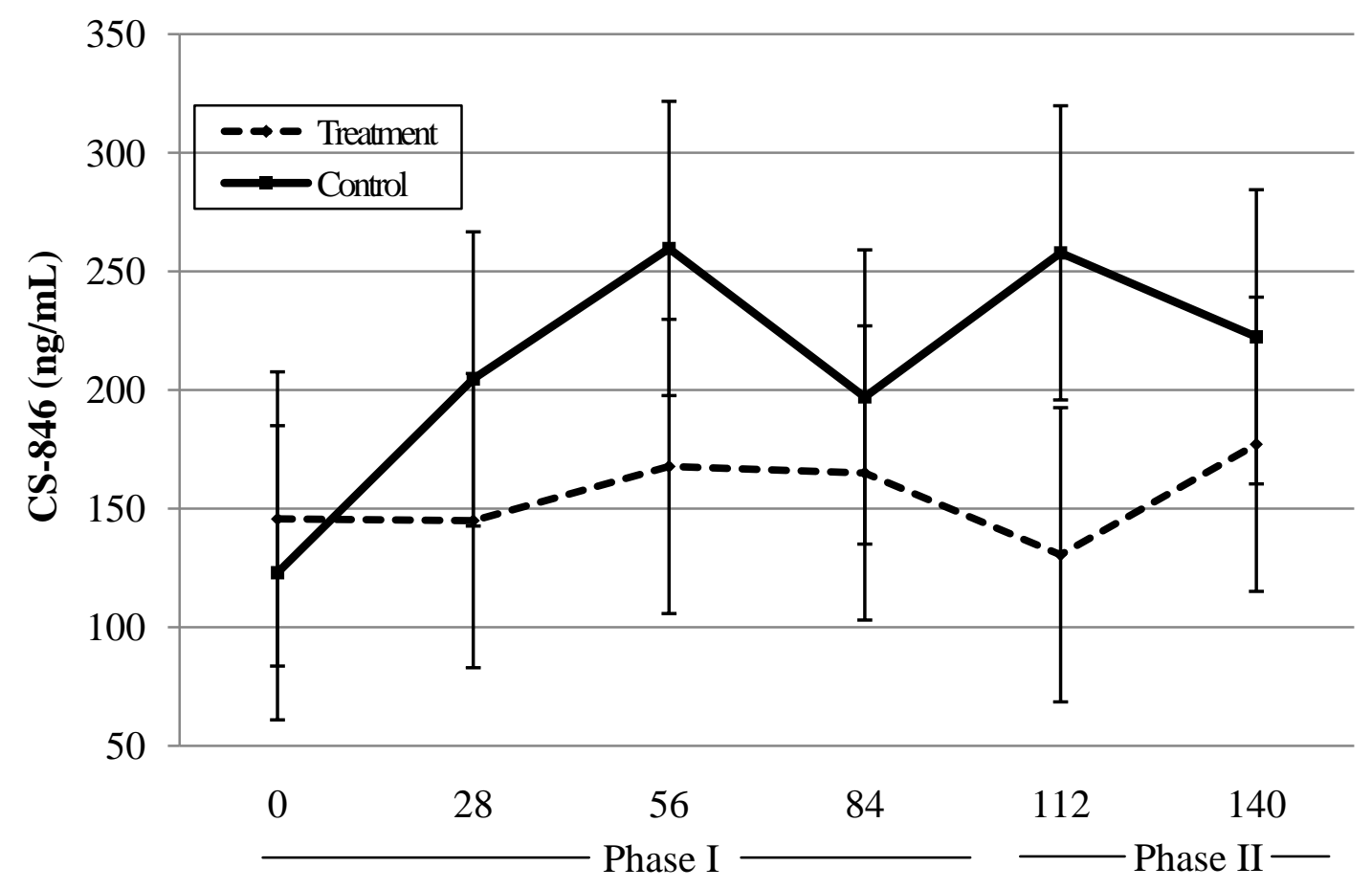

Time (d)

Figure 7. Serum concentrations (ng/mL) of the epitope chondroitin sulfate 846 (CS-846) over time (d) beginning with phase I (d 0 to 110) consisting of ground work and early training to phase II (d 111-140) representing moderate workload and application of advanced maneuvers in horses receiving a pelleted concentrate with (Treatment) or without (Control) supplementation of a marine based omega 3 product.

The initial increase in concentrations of CS-846 on d 56 is most likely a reflection of bone remodeling in response to exercise. Nielson et al. (1997) reported the remodeling of bone occurs approximately every 62 to $104 \mathrm{~d}$ in order to accommodate new forces placed upon the joint as a result of increases in workload. In this study, the exercise protocol leading up to $\mathrm{d} 56$ included the introduction of advanced maneuvers (lope departures, loping circles, and hindquarter pivots), which would correspond to changes in forces placed upon the joint, and therefore would have the potential to induce 
remodeling on approximately d 56. During remodeling, bone is at its weakest. In order to compensate, type II collagen and aggrecan synthesis increases during bone remodeling in order to more effectively distribute mechanical load during exercise (McIlwraith and Trotter, 1996). Therefore, increased synthesis of these molecules would be reflected by increased concentrations of CS-846 in synovial fluid, resulting in the release of these small peptide fragments into circulation.

Initial concentrations of CPII and CS-846 were similar $(<1000 \mathrm{ng} / \mathrm{mL}$, respectively) to a previous study utilizing $2 \mathrm{yr}$ old horses that were free of lameness and were considered to have radiographically normal carpal joints (Frisbie et al., 2008). Horses were exercised on a high-speed treadmill to simulate strenuous exercise race training that consisted of galloping $(32 \mathrm{~km} / \mathrm{h})$ and trotting $(16-19 \mathrm{~km} / \mathrm{h})$.

The increasing serum concentrations of CPII and CS-846 in response to exercise in the current study are similar to those observed by Frisbie et al. (2008). However, they observed an increase in serum concentrations in exercised horses over time without changes in exercise intensity or duration. Similarities between the current study and the results of Frisbie et al. (2008) indicate that the workload used in the current study was strenuous enough to induce changes in articular cartilage. While the current study did not evaluate concentrations of CPII and CS-846 in synovial fluid, Frisbie et al. (2008) determined that mean levels of CPII and CS-846 were 50\% and 20-fold higher, respectively, within synovial fluid when compared to serum.

Furthermore, increased synthesis of type II collagen and aggrecan molecules has also been associated with the early stages of joint disease (McIlwraith and Trotter, 
1996). The development of OA is considered to progress more rapidly in horses when compared to humans (Frisbie et al., 2008). Increased serum concentrations of CPII and CS-846 in the present study may indicate a reparative response. Frisbie et al. (1999) reported increases in serum CPII and CS-846 in response to grade 0 to 2 of osteochondral fragmentation scores however, horses with scores of 3 or higher had lower concentrations of these epitopes, which may potentially indicate an inability of the cartilage to carry out reparative processes. Therefore, these markers have the potential to detect damage in the early stages of OA when the joint is still capable of reparative processes before entering in a degenerative state.

\section{Synovial Fluid Inflammatory Markers}

Omega 3 fatty acid supplementation did not influence concentrations of WBC, TP, or SG $(\mathrm{P}=0.47, \mathrm{P}=0.52$, and $\mathrm{P}=0.51$, respectively; Table 4). However, specific gravity of synovial fluid increased over time $(\mathrm{P}<0.001)$ in response to increased intensity and duration of exercise. However, there was no influence of time on WBC or $\mathrm{TP}(\mathrm{P}=0.11$ and $\mathrm{P}=0.16)$.

Table 4. Least square treatment means of white blood cell count (WBC), total protein (TP), and specific gravity (SG) in synovial fluid of horses with (Treatment) or without (Control) omega 3 supplementation

\begin{tabular}{lcrrc}
\hline \hline Parameter & Treatment $^{1}$ & Control & SEM & $P$-value \\
\hline WBC,$\mu \mathrm{L}$ & 121.46 & 136.94 & 14.74 & 0.47 \\
TP, g/dL & 1.31 & 1.15 & 0.17 & 0.52 \\
SG & 1.01 & 1.01 & 0.01 & 0.51 \\
\hline
\end{tabular}

${ }^{1}$ Addition of $350 \mathrm{~g} / \mathrm{d}$ of a commercial marine-based omega 3 fatty acid supplement

${ }^{2}$ Effect of dietary treatment on parameters 
Indirect markers (WBC, TP, and SG) contained within synovial fluid are indicative of cytokine and eicosanoid production, and have been reported to increase with joint damage. This relationship makes them useful indicators of joint inflammation (Bertone et al., 2001). Inflammation is considered to be the body's immediate response to infection, increasing the permeability across capillaries to allow for large molecules to leave the bloodstream and enter into the surrounding plasma (Calder, 2002).

The current study observed an increase in SG concentrations or cellular debris (including leukocytes and monocytes) entering synovial fluid in response to exercise. In a previous study, SG concentrations were also shown to increase when horses entered into training (Persson et al., 1971). In contrast, TP remained within a normal range (< $2.5 \mathrm{~g} / \mathrm{dl}$ ) throughout the trial, indicating non-infective inflammatory conditions within the joint (McIlwraith et al., 1987).

The synovial WBC concentration represents the health of the joint and joint fluid, and is not greatly affected by other inflammatory process occurring within the joint (Bertone et al., 2001). McIlwraith and Trotter (1996) concluded that the quantitative and qualitative changes in leukocytes can provide an indication of the magnitude of synovial membrane inflammation. Bertone et al. (2001) reported synovial WBC correlated highly with IL-6 concentrations, which is considered to be an excellent predictor of joint disease. Concentrations of IL-6 were the most sensitive and specific for joint disease and could potentially be utilized as a screening test for the presence of joint disease when lameness is difficult to identify (Bertone et al., 2001). Therefore, the use of WBC can be considered a useful indicator of joint disease. 
Gibson et al. (1996) had reported higher synovial WBC in horses affected with joint disease when compared to normal joints (183 \pm 142 vs. $112 \pm 189$ cells $/ \mu \mathrm{L})$. However, values were not different due to high individual variations. McIlwraith (1987) and Van Pelt (1974) determined similar concentrations in horses diagnosed with chronic degenerative disease. A previous study in our laboratory, utilizing an arthritic model, determined the addition of n-3 supplementation decreased synovial fluid WBC in supplemented horses when compared to control (Manhart et al., 2009). The WBC concentrations were greater when compared to values observed in this study (41 to 5750 cells $/ \mathrm{mL}$ vs. 0.015 to 0.368 cells $/ \mathrm{mL}$ ), due to their arthritic state.

The difference in models (young vs. arthritic) and degree of joint inflammation may explain the conflicting results observed. It is also important to note that synovial fluid WBC, TP, and SG values remained within normal values despite repeated arthrocentesis every $28 \mathrm{~d}$ of the study. While the procedure itself induces an inflammatory response, van den Boom et al. (2005) concluded that while repeated arthrocentesis is a confounding factor in trials of this nature, the effect can be avoided if 1 or more wk separates the procedures. Perhaps these inflammatory markers (WBC, TP, and SG) are not sensitive enough for use in preventative studies of this nature. 


\section{CHAPTER V}

\section{SUMMARY}

Previous studies have suggested the potential of n-3 FA supplementation to mitigate inflammation associated with horses previously diagnosed with OA. However, little information exists on measuring the effectiveness of $n-3$ supplementation in an exercising, non-arthritic model. As performance characteristics are difficult to measure objectively, the potential use of anabolic markers relative to collagen synthesis could be utilized as a non-invasive means to evaluate the effect of $n-3$ supplementation on joint health as well as the effect of changes to the joint in response to exercise intensity and duration.

This study indicated n-3 supplementation did not affect synovial WBC, TP, or SG. In addition, there was no influence of time on WBC and TP, although, the SG of synovial fluid increased over time when transitioning from phase I to phase II of the experiment. Dietary treatment did not influence serum concentrations of CPII or CS846. However, CS-846 tended to increase over time and CPII concentrations also increased over time as exercise duration and intensity increased. Additionally, all horses gained BW and BCS throughout the trial, but there was no difference in BW or BCS with treatment.

Based on these results, further studies are needed to determine the efficacy of n-3 supplementation as a preventive measure for young horses in training against articular cartilage damage that can lead to OA later in life. Current treatment for OA focuses 
primarily on controlling pain, and many of these medications have undesirable side effects, and therefore determining a preventative measure in the form of a dietary product would be advantageous. 


\section{LITERATURE CITED}

Benito, M. J., D. J. Veale, O. Fitzgerald, W. B. van den Berg, B. Bresnihan. 2005. Synovial tissue inflammation in early and late osteoarthritis. Ann. Rheum. Diseases 64:1263-1267.

Bertone, A. L., J. L. Palmer, and J. Jones. 2001. Synovial fluid cytokines and eicosanoids as markers of joint disease in horses. Vet. Surg. 30:528-531.

Bierer, T. L. and L. M. Bui. 2002. Improvement of arthritic signs in dogs fed greenlipped mussel. J. Nutr. 132:1634-1636.

Billinghurst, R. C., P. J. Brama, P. R. van Weeren, M. S. Knowlton, and C. W. McIlwraith. 2003. Significant exercise-related changes in the serum levels of two biomarkers of collagen metabolism in young horses. Osteoarthritis and Cartilage 11:760-769.

Brama, P. J., J. M. TeKoppele, R. A. Bank, A. Barneveld, and P. R. van Weeren. 2000. Functional adaptation of equine articular cartilage: the formation of regional biochemical characteristics up to age one year. Equine Vet. J. 32:217-221.

Brama, P. J., J.M. TeKoppele, R. A. Bank, A. Barneveld, and P. R. van Weeren. 2002. Development of biochemical heterogeneity of articular cartilage: influences of age and exercise. Equine Vet. J. 34(3):265-269.

Brilla, L. B., and T. E. Landerholm. 1990. Effect of fish oil supplementation and exercise on serum lipids and aerobic fitness. J. Sports Med. Phys. Fit. 30:173180.

Budsberg, S. C., S. A. Johnston, P. D. Schwarz, C. E. Decamp, and R. Claxton. 1999. Efficacy of etodolac for the treatment of osteoarthritis of the hip joints in dogs. J. Amer. Med. Assoc. 214:206-210.

Burdge, G. 2004. Alpha-linolenic acid metabolism in men and women: nutritional and biological implications. Curr. Opin. Clin. Nutr. Metab. Care 7:137-144.

Calder, P. C. 2002. Dietary modification of inflammation with lipids. Proc. Nutr. Soc. 61:345-358.

Caterson, B., C. R. Flannery, C. E. Hughes, and C. B. Little. 2000. Mechanisms involved in cartilage proteoglycan catabolism. Matrix Biol. 333-344. 
Caughey, G. E., M. Pouliot, L. G. Cleland, and M. G. James. 1997. Regulation of tumor necrosis factor alpha and interleukin-1 beta synthesis by thromboxane $\mathrm{A}_{2}$ in nonadherent human monocytes. J. Immunol. 158:351-358.

Christenson, J. H., M. S. Christenson, J. Dyenberg, and E. B. Schmidt. 1999. Heart rate variability and fatty acid content of blood cell membranes: a dose dependent study with n-3 fatty acids. Am. J. Clin. Nutr. 70:331-337.

Cleland, L. G., J. K. French, W. H. Betts. 1988. Clinical and biochemical effects of dietary fish oil supplements in rheumatoid arthritis. J. Rheum. 15:1471-1475.

Cleland, L. G., M. J. James, and S. M. Proudman. 2003. The role of fish oils in the treatment of rheumatoid arthritis. Drugs 63(9): 845-853.

Conte, A., M. de Bernardi, L. Palmieri, P. Lualdi, G. Mautone, and G. Ronca. 1991. Metabolic fate of exogenous chondroitin sulfate in man. Arzneimittelforschung 41(7):768-772.

Curtis, C. L., C. E. Hughes, C. R. Flanner, C. B. Little, J. L. Harwood, and B. Caterson. 2000. N-3 fatty acids specifically modulate catabolic factors involved in articular cartilage degradation. J. Biol. Chem. 275(2):721-725.

Curtis, C. L., S. G. Rees, C. B. Little. 2002. Pathological indicators of degradation and inflammation in human osteoarthritic cartilage are abrogated by exposure of n-3 fatty acids. Arthritis Rheum. 46:1544-1553.

Das, U.N. 2000. Beneficial effect(s) of n-3 fatty acids in cardiovascular diseases: but, why and how? Prostaglandins, Leukotrienes and Essential Fatty Acids 63:351262.

de Lorgeril M., S. Renaud, N. Mamelle, P. Salen, J. L. Martin, I. Monjaud, J. Guidollet, P. Touboul, and J. Delaye. 1994. Mediterranean alpha-linolenic acid-rich diet in secondary prevention of coronary heart disease. Lancet 343:1454-1459.

Dijkgraaf, L. C., L. G. Debont, G. Boering, and R. S. Liem. 1995. Normal cartilage structure, biochemistry, and metabolism: a review of the literature. J. Oral. Maxillofac. Surg. 53:924-929.

Duncan, R. L. and C. H. Turner. 1995. Mechanotransduction and the functional response of bone and mechanical strain. Calc. Tissue Int. 57:344-358.

Dyenberg, J. and H. O. Bang. 1979. Haemostatic function and platelet polyunsaturated fatty acids in Eskimos. Lancet 2:433-435. 
Dyenberg, J., H. Bang, and N. Hjorne. 1975. Fatty acid composition of the plasma lipids in Greenland Eskimos. Acta. Med. Scand. 28:85-94.

Dyenberg, J., H. Bang, and E. Stofferson. 1978. Eicosapentaenoic acid and prevention of thrombosis and atherosclerosis. Lancet 2: 117-119.

Frisbie, D. D., F. Al-Sobayil, R. C. Bilinghurst, C. E. Kawcak, and C.W. McIlwraith. 2008. Changes in synovial fluid and serum biomarkers with exercise and early osteoarthritis in horses. Osteoarthritis and Cartilage 16:1196-1204.

Frisbie, D. D., C. S. Ray, M. Ionescu, A. R. Poole, P. L. Chapman, and C. W. McIlwraith. 1999. Measurement of the 846 epitope of chondroitin sulfate and of carboxy propeptides of type II procollagen for diagnosis of osteochondral fragmentation in horses. Am. J. Vet. Res. 60:306-309.

Glant, T. T., M. Katalin, P. J. Roughley, E. Buzas, and A. R. Poole. 1986. Age-related changes in protein-related epitopes of human articular-cartilage proteoglycans. Biochem. J. 236:71-75.

Gibson, K. T., H. Hodge, and T. Whittem. 1996. Inflammatory mediators in equine synovial fluid. Australian Vet. J. 73(4):148-151.

Goldberg, R. J. and J. Katz. 2007. A meta-analysis of the analgesic effects of omega-3 polyunsaturated fatty acid supplementation for inflammatory joint pain. Pain 129:210-223.

Goodrich, L. R., and A. J. Nixon. 2006. Medical treatment of osteoarthritis in the horse-a review. Vet. J. 171:51-69.

Hansen, R. A., C. J. Savage, K. Reidlinger, J. L. Traub-Dargatz, G. K. Ogilvie, D. Mitchell, and M. J. Fettman. 2002. Effects of dietary flaxseed oil supplementation on equine plasma fatty acid concentrations and whole blood platelet aggregation. J. Vet. Intern. Med. 16:457-463.

He, K., Y. Song, M. Daviglus, K. Liu, L. Van Horn, A. Dyer, and P. Greenland. 2004. Accumulated evidence on fish consumption and coronary heart disease mortality: a meta-analysis of cohort studies. Circulation 109:2705-2711.

Helminen, H.J., M. M. Hytinen, M. J. Lammi, J. P. Arokoski, T. Lepvetelainen, and T. Jurvelin. 2000. Regular joint loading in youth assists in the establishment and strengthening of the collagen network of articular cartilage and contributes to the prevention of osteoarthritis later in life: a hypothesis. J. Bone Miner. Metab. 18(5):245-257. 
Henneke, D. R., G. D. Potter, J. L. Kreider, and B. F. Yeates. 1983. Relationship between condition score, physical measurements and body fat percentage in mares. Equine Vet. J. 15:371-372.

Hjalmarson, A., E. A. Gilpin, J. Kjekshus. 1990. Influence of heart rate on mortality after acute myocardial infarction. Am. J. Cardiol. 65:547-553.

Holland, J. L., D. S. Kronfeld, G. A. Rich, K. A. Kline, J. P. Fontenot, T. N. Meacham, and P. A. Harris. 1997. Acceptance of fat and lecithin containing diets by horses. Appl. Anim. Behav. Sci. 56:91-96.

Hodgson, D. R., R. J. Rose, T. B. Kelso, L. J. McCutcheon, W. M. Bayly, and P. D. Gollnick. 1990. Respiratory and metabolic responses in the horse during moderate and heavy exercise. Pflugers Arch. Eur. J. Nutr. 60:217-224.

Jee, W.S. 1988. The skeletal tissues. Pages 207-255 in Histology: Cell and Tissue Biology, $5^{\text {th }}$ ed. L. Weiss, ed. Elsevier Biomedical, New York, NY.

Jeffcoat, L. B., P. D. Rossdale, and J. Freestone. 1982. An assessment of wastage in Thoroughbred racing from conception to 4 years of age. Equine Vet J. 14:185198.

Julen, T. T., G. D. Potter, L. W. Green, and G. G. Stott. 1995. Adaptation to a fatsupplemented diet by cutting horses. Pages 56-61 in Proc. of the $14^{\text {th }}$ ENPS, Ontario, CA.

Jurvelin, J., I. Kiviranta, M. Tammi, and J. H. Helminen. 1986. Softening of the canine articular cartilage after immobilization of the knee joint. Clin. Orthop. 207:246252.

King, S. S., K. L. Jones, G. A. Apgar, and A. AbuGhazaleh. 2005. Fatty acid profiles from red blood cells in response to three levels of dietary omega-3 fatty acid supplementation. Pages 364-365 in Proc. of the $19^{\text {th }}$ Equine Sci. Soc., Tucson, AZ.

King, S. S., A. AbuGhazaleh, S. K. Webel, and K. L. Jones. 2008. Circulating fatty acids profiles in response to three levels of dietary omega-3 supplementation fatty acid supplementation in horses. J. Anim. Sci. 86:1114-1123.

Kris-Etherton, P. M., W. Harris, and L. Appel. 2002. Fish consumption, fish oil, omega3 fatty acids, and cardiovascular disease. Circulation 106:2747-2757. 
Kiviranta, I., M. Tammi, and J. Jurvelin. 1988. Moderate running exercise augments glycosaminoglycans and thickness of articular cartilage in the knee joint of beagle dogs. J. Ortho. Res. 6:188-195.

Li B., C. Birdwell, and J. Whelan. 1994. Antithetic relationship of dietary arachidonic acid and eicosapentaenoic acid on eicosanoid production in vivo. J. Lipid Res. $35: 1869-1877$.

Leaf, A., J. X. Kang, Y. F. Xiao, and G. E. Billman. 2003. Clinical prevention of sudden cardiac death by $n-3$ polyunsaturated fatty acids and mechanism of prevention of arrhythmias by n-3 fish oils. Circulation 107:2646-2652.

Lohmander, L. S., H. Roos, L. Dahlberg. 1994. Temporal patterns of stromelysin-1, tissue inhibitor, and proteoglycan fragments in human knee joint fluid after injury to the cruciate ligament or meniscus. J. Orth. Res. 12:21-28.

MacAllister, C. G., S. J. Morgan, A. T. Borne, R. A. Pollet. 1993. Comparison of adverse effects of phenylbutazone, flunixin meglumin, and ketoprofen in horses. J. Am. Vet. Med. Assoc. 202(1):71-77.

Manhart, D.R., B. D. Scott, E. M. Eller, C. M. Honnas, D. M. Hood, J. A. Coverdale, and P. G. Gibbs. 2009. Effect of n-3 PUFAs on markers of inflammation in arthritic horses. Prof. Anim. Sci. 25:155-160.

Marcel, Y. L., K. Christiansen, and R. T. Holman. 1968. The preferred metabolic pathway from linoleic acid to arachidonic acid in vitro. Biochim. Biophys. Acta. $164: 25-34$.

Marsh, J. B., D. L. Topping, and P. J. Nestel. 1987. Comparative effects of dietary fish oil carbohydrate on plasma lipids and hepatic activities of phosphatidase phosphohydrolase, diacylglycerol acyltransferase and neutral lipase activites in the rat. Biochim. Biophys. Acta. 922:239-243.

May, S. A., R. E. Hooke, and P. Lees. 1992. Interleukin-1 stimulation of equine articular cells. Res. Vet. Sci. 52:342-348.

McIlwraith, C. W. 1987. Diseases of joints, tendons, ligaments, and related structures. Page 339 in Adams Lameness in Horses. T. S. Stashak, ed. Lea and Febiger, Philadelphia, PA.

McIlwraith, C. W. and G. W. Trotter. 1996. Joint Disease in the Horse. W.B. Saunders Co. Philadelphia, PA. 
Meyers, M. C., G. D. Potter, L. W. Greene, S. F. Crouse, and J. W. Evans. 1989. Physiologic and metabolic response of exercising horses to added dietary fat. J. Equine Vet. Sci. 9(4):218-223.

Munsterman, A. S., A. L. Bertone, T. A. Zachos, and S. E. Weisbrode. 2005. Effects of the omega-3 fatty acid, alpha-linolenic acid on lipopolysaccharide-challenged synovial explants from horses. Am. J. Vet. Res. 66:1503-1508.

Nakamura, M. T. and T. Y. Nara. 2002. Essential fatty acid synthesis and its regulation in mammals. Prostaglandins, Leukotrienes and Essential Fatty Acids 64:145-150.

Neil, K. M., J. P. Caron, and M. W. Orth. 2005. The role of glucosamine and chondroitin sulfate in treatment for and prevention of osteoarthritis in animals. J. Am. Vet. Med. Assoc. 226:1079-1088.

Nelson, F. A. Reiner, and M. Ionescu. 1994. The control of C-propeptide of type II collagen in articular cartilage is an index of synthesizing this molecule which is increased in osteoarthritis. Pages 216-237 in Proc. of the $40^{\text {th }}$ Annual Meeting of the Orthopedic Research Society, New Orleans, LA.

Newton, I. S. 1996. Long chain fatty acids in health and nutrition. J. Food Lipids 3:233249.

Nielson, B.D., G. D. Potter, E. L. Morris, M. Murray-Gerzik, W. B. Smith, and M. T. Martin. 1997. Changes in the third metacarpal bone and frequency of bone injuries in young Quarter Horses during race training-observations and theoretical considerations. J. Equine Vet. Sci. 17:541-549.

Nilsson, B. E. and N. E. Westlin. 1971. Bone density in athletes. Clin. Ortho. 77:179182.

Norwood, G. L. 1978. The bucked shin complex in thoroughbreds. Pages 319-336 in Proc. Am. Assoc. Equine Pract., St. Louis, MO.

NRC, 2007. Nutrient Requirements of Horses. $6^{\text {th }}$ rev. ed. Natl. Acad. Press, Washington, DC.

Obata T., T. Nagakura, T. Masaki, K. Maekawa, and K. Yamashita. 1999. Eicosapentaenoic acid inhibits prostaglandin $\mathrm{D}_{2}$ generation by inhibiting cyclooxygenase-2 in cultured human mast cells. Clinical and Experimental Allergy 29:1129-1135. 
O’Connor, C. I., A. C. St. Lawrence, L. M. Lawrence. 2001. The effect of dietary omega-3 fatty acids on the fatty acid profile of equine serum. Pages 437-438 in Proc. of the $17^{\text {th }}$ ENPS, Lexington, KY.

O’Connor, C. I. and L. M. Lawrence. 2004. The effect of dietary fish oil supplementation in exercising horses. J. Anim. Sci. 82:2978-2984.

O’Connor, C. I., L. M. Lawrence, and S. H. Hayes. 2007. Dietary fish oil supplementation affects serum fatty acid concentrations in horses. J. Anim. Sci. 85:2183-2189.

O’Connor, P., C. R. Orford, and D. L. Gardner. 1988. Differential response to compressive loads of zones of canine hyaline articular cartilage: micromechanical light and electron microscope studies. Ann. Rheum. Dis. 47:414-420.

O'Keefe, J. H., H. Abruissa, A. Sastre, D. M. Steinhaus, and W. S. Harris. 2006. Effects of omega-3 fatty acids on resting heart rate, heart rate recovery after exercise, and heart rate variability in men with healed myocardial infarctions and depressed ejection fractions. Am. J. Cardiol. 97: 1127-1130.

Palmer, R. J. and A.L. Bertone. 1994. Joint structure, biochemistry and biochemical disequilibrium of synovitis and equine joint disease. Equine. Vet J. 26:263-277.

Persson, L. 1971. On the synovia in horses: a clinical and experimental study. Acta. Vet. Scand. Suppl. 35:1-77.

Poole, R.A., G. Rizkalla, and A. Reiner. 1992. Changes in the extracellular matrix of articular cartilage in human osteoarthritis. Pages 3-12 in Trends in Research and Treatment of joint diseases, Hirohata K. and T. Matsubara, ed. Springer-Verlag, New York.

Poole, R. A., M. Ionsecu, A. Swan, and P. Dieppe. 1994. Changes in cartilage metabolism in arthritis are reflected by altered serum and synovial fluid levels of glycosaminoglycan epitopes on fragments of the cartilage proteoglycan aggrecan: implications for pathogenesis. J. Clin. Invest. 94:25-33.

Potter, G. D., S. P. Webb, J. W. Evans, and G. W. Webb. 1990. Digestible energy requirements for work and maintenance of horses fed conventional and fatsupplemented diets. J. Equine Vet. Sci. 10(3):214.

Rizkalla, G., A. Reiner, E. Bogoch, and A. R. Poole. 1992. Studies of the articular cartilage proteoglycan aggrecan in health and osteoarthritis. Evidence for molecular heterogeneity and extensive molecular changes in disease. J. Clin. Invest. 90:2268-2277. 
Rohde, T. D. A. MacLean, E. A. Richter, B. Kiens, and B. K. Pederson. 1997. Prolonged submaximal eccentric exercise is associated with increased levels of plasma IL-6. Am. J. Physiol. 273:85-91.

Ross, T. N., G. D. Potter, E.M. Eller, B. D. Scott, P. G. Gibbs, and H. W. Sampson. 2007. Evaluation of inflammatory markers in yearlings fed various sources of polyunsaturated fatty acids. Pages $104-105$ in Proc. of the $20^{\text {th }}$ Equine Sci. Soc., Baltimore, MD.

Rossdale, P. D., R. Hopes, N. J. Wingfield-Digby. 1985. Epidemiological study of wastage among racehorses 1982 and 1983. Vet. Rec. 116:66-69.

Roudebush, P. 2008. Pain management for canine osteoarthritis. Pages 1007-1010 in Proc. N. Amer. Vet. Conf., Orlando, FL.

Samuelsson, B., S. E. Dahlen, J. A. Lindhren, C. A. Rouzer, and C. N. Serhan. 1987. Leukotrienes and lipoxins: structures, biosynthesis, and biological effects. Science 237:1171-1176.

Sandell, L. J. and T. Aigner. 2001. Review: articular cartilage and changes in arthritis an introduction: cell biology of osteoarthritis. Arthritis Res. 3:107-113.

Saynor, R. and T. Gillott. 1992. Changes in blood lipids and fibrinogen with a note on safety in long term study on the effects of n-3 fatty acids in subjects receiving fish oil supplements and followed for seven years. Lipids 27:1283-1291.

Scott, B. D., G. D. Potter, L. W. Greene, P. S. Harris, and J. G. Anderson. 1992. Efficacy of a fat-supplemented diet on muscle glycogen concentrations in exercising thoroughbred horses maintained in varying body conditions. J. Equine Vet. Sci. 12:109-113.

Shinmei, M., Y. Yoshihara, and K. Iwata. 1992. Stromelysin and TIMP levels in joint fluid and serum from the patients of joint diseases. Page 62 in Proc. of the $38^{\text {th }}$ meeting of the Ortho. Res. Soc., Washington, DC.

Simopoulos, A. P. 2002. The importance of the ratio of omega-6/omega-3 essential fatty acids. Biomed. Pharma. 56:365-379.

Smith, R. K., H. Birch, J. Patetterson-Kane, E. C. Firth, L. Williams, W. Cherdchutham, W. R. van Weeren, and A. E. Goodship. 1999. Should equine athletes commence training during skeletal development?: changes in tendon matrix associated with development, ageing, function, and exercise. Equine Vet. J. 31:201-209. 
Surette, M.E., J. Wheelan, G. P. Lu, K.. S. Broughton, and J. E. Kinsella. 1992. Dependence on dietary cholesterol for n-3 polyunsaturated fatty acid-induced changes in plasma cholesterol in the Syrian hamster. J. Lipid Res. 30: 263-271.

Todhunter, R. J. and G. Lust. 1990. Pathophysiology of synovitis: clinical signs and examination in horses. Compendium on Continuing Edu. Pract. Vet. 12:980-992.

Vance, D. E. and J. E. Vance. 1991. Biochemistry of Lipids, Lipoproteins, and Membranes. Elsevier, New York, NY.

Vane, J. R., Y. S. Bakhle, and R. M. Botting. 1998. Cyclooxygenases 1 and 2. Annual Review of Pharm. and Toxicology 38:97-120.

van den Boom, R., C. H. van de Lest, S. Bull, R. A. Brama, P. R. van Weeren, and A. Barneveld. 2005. Influence of repeated arthrocentesis and exercise on synovial fluid concentrations of nitric oxide, prostaglandin E2 and glycosaminoglycans in healthy equine joints. Equine Vet. J. 37:250-256.

Van Pelt, R. W. 1974. Interpretation of synovial fluid findings in the horse. J. Amer. Vet. Med. Assoc. 165:91-95.

van Weeren, P. R., E. C. Firth, H. Brommer, M. Hyttinen, H. J. Helminen, C. W. Rogers, J. DeGroot, and P. J. Brama. 2008. Early exercise advances the maturation of glycosaminoglycans and collagen in the extracellular matrix of articular cartilage in the horse. Equine Vet. J. 40:128-135.

Webb, S. P., G. D. Potter, and J. W. Evans. 1987. Physiologic and metabolic response of race and cutting horses to added dietary fat. Page 115 in Proc. of the $10^{\text {th }}$ ENPS, Ft. Collins, CO.

Westacott, C. I. and M. Shariff. 1996. Cytokines in osteoarthritis: Mediators or markers of joint destruction? Semin. Arthritis Rheum. 25:254-272.

Woodward, A. D., B. D. Nielson, C. I. O’Connor. S. K. Webel, and M. W. Orth. 2005. Dietary long chain polyunsaturated fatty acids increase plasma eicosapentaenoic acid and docosahexaenoic acid concentrations and trot stride length in horses. Pages 101-106 in Proc. $19^{\text {th }}$ Equine Sci. Soc., Tucson, AZ.

Zurier, R. B., R. G. Rossetti, S. H. Burstein, and B. Bidinger. 2003. DimethylheptylTHC-11 oic acid: A non-psychoactive anti-inflammatory agent in cannabinoid template structure. Arthritis Rheum. 41:163-170. 


\section{VITA}

Name: Jessica Lauren Lucia

Address: $\quad 2471$ TAMU

College Station, TX 77843-2471

Email Address: jllucia@tamu.edu

Education: $\quad$ B.S., Animal Science, Texas A\&M University, 2007

M.S., Animal Science, Texas A\&M University, 2009 